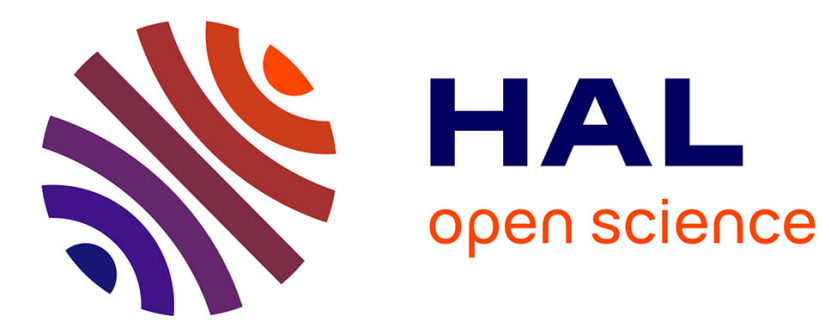

\title{
Gröbner Bases, Symbolic Summation and Symbolic Integration
}

\author{
Frédéric Chyzak
}

\section{To cite this version:}

Frédéric Chyzak. Gröbner Bases, Symbolic Summation and Symbolic Integration. [Research Report] RR-3297, INRIA. 1997. inria-00073391

\section{HAL Id: inria-00073391 \\ https://hal.inria.fr/inria-00073391}

Submitted on 24 May 2006

HAL is a multi-disciplinary open access archive for the deposit and dissemination of scientific research documents, whether they are published or not. The documents may come from teaching and research institutions in France or abroad, or from public or private research centers.
L'archive ouverte pluridisciplinaire HAL, est destinée au dépôt et à la diffusion de documents scientifiques de niveau recherche, publiés ou non, émanant des établissements d'enseignement et de recherche français ou étrangers, des laboratoires publics ou privés. 
INSTITUT NATIONAL DE RECHERCHE EN INFORMATIQUE ET EN AUTOMATIQUE

\section{Gröbner Bases, Symbolic Summation and Symbolic Integration}

Frédéric CHYZAK

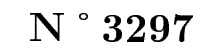

Novembre 1997

THÈME 2

\section{apport}





\title{
Gröbner Bases, Symbolic Summation and Symbolic Integration
}

\author{
Frédéric CHYZAK \\ Thème 2 - Génie logiciel \\ et calcul symbolique \\ Projet Algo
}

Rapport de recherche $\mathrm{n}^{\circ} 3297$ - Novembre 1997 - 31 pages

\begin{abstract}
The treatment of combinatorial expressions and special functions by linear operators is amenable to Gröbner basis methods. In this tutorial, we illustrate the applications of Gröbner bases to symbolic summation and integration.
\end{abstract}

(Résumé : tsvp)

Unité de recherche INRIA Rocquencourt

Domaine de Voluceau, Rocquencourt, BP 105, 78153 LE CHESNAY Cedex (France)

Téléphone : (33) 0139635511 - Télécopie : (33) 0139635330 


\section{Bases de Gröbner, Sommation symbolique et Intégration symbolique}

Résumé : De nombreuses méthodes de manipulation d'expressions combinatoires et de fonctions spéciales au moyen d'opérateurs linéaires se fondent sur le calcul de bases de Gröbner. Dans cet exposé introductif, nous illustrons

les applications des bases de Gröbner dans le domaine de la sommation et de l'intégration symboliques. 


\title{
Gröbner Bases, Symbolic Summation and Symbolic Integration
}

\section{Frédéric Chyzak}

INRIA-Rocquencourt and École polytechnique (FRANCE)

Frederic.Chyzak@inria.fr

\begin{abstract}
The treatment of combinatorial expressions and special functions by linear operators is amenable to Gröbner basis methods. In this tutorial, we illustrate the applications of Gröbner bases to symbolic summation and integration.
\end{abstract}

\section{Introduction}

In the late 1960's, Risch $(1969,1970)$ developed an algorithm for symbolic indefinite integration. The approach followed there consists in computing a tower of differential extensions in order to determine if an indefinite integral can be expressed in terms of elementary functions. Risch's algorithm became very popular and is now at the heart of the integration routines of many computer algebra systems. In the early 1980's, Karr $(1981,1985)$ appealed to similar ideas, namely difference extensions, in order to develop an algorithm for symbolic indefinite summation. Despite its indisputable algorithmic interest, Karr's algorithm has unfortunately not received as much attention as it deserves yet, due to its complexity and the difficulty to implement it.

In the early 1990's, Zeilberger (1990b) initiated a different approach to symbolic summation and integration. As opposed to the approach by differential or difference extensions, Zeilberger studies the action of algebras of differential or difference linear operators in order to compute special operators that determine the sum or integral under consideration. The method is based on the theory of holonomy (Bernstein 1971, 1972; Björk 1979; Coutinho 1995; Ehlers 1987; Kashiwara 1978). Since then, Zeilberger has improved his first approach so much that his "fast algorithm" is the basis for the definite summation routines of many computer algebra systems (Petkovšek et al. 1996; Wilf and Zeilberger 1992a, 1992b; Zeilberger 1990a).

More specifically, the starting point of the operator approach is to consider which linear operators annihilate a given function or sequence. A function, respectively a sequence, is then described by a set of annihilating operators. 
For example, the binomial coefficients $\left(\begin{array}{c}n \\ j\end{array}\right)$ satisfy linear recurrence equations. Let $S_{n}$ denote the operator of shift with respect to $n$ : this operator acts on a function $f$ by $\left(S_{n} \cdot f\right)(n)=f(n+1)$. Shifts with respect to other variables are denoted analogously. Identifying a rational function $r$ in $(n, j)$ with the linear operator of multiplication by $r$, we get a non-commutative algebra whose product denotes the composition of operators. With this notation, the binomial coefficients are annihilated by each operator of the system

$$
(n+1-j) S_{n}-(n+1), \quad(j+1) S_{j}-(n-j) .
$$

These operators correspond to the vertical and horizontal recurrences in Pascal's triangle. Summation is then recast into a different form: informally, the left ideal spanned by the previous operator system encodes all the linear equations with rational function coefficients satisfied by the binomial coefficients; summation then reduces to deducing an operator of a special form starting from the description (0.1) of the ideal. More precisely, the summation over $j$ requires an operator which does not involve $j$, i.e., an operator in $n, S_{n}$ and $S_{j}$ only. For instance, deriving Pascal's triangle rule, as encoded by the operator $P=S_{n} S_{j}-S_{j}-1$, suffices to perform the summation over $j$. This derivation is based on the elimination of $j$, for which methods are detailed in the main sections of this tutorial. A rewritten form for $P$ is $\left(S_{j}-1\right)\left(S_{n}-1\right)+\left(S_{n}-2\right)$, which applied to $\left(\begin{array}{l}n \\ j\end{array}\right)$ yields

$$
g_{n, j+1}-g_{n, j}+\left[\left(S_{n}-2\right) \cdot f\right](n, j)=0
$$

for $f_{n, j}=\left(\begin{array}{c}n \\ j\end{array}\right)$ and $g_{n, j}=\frac{j}{n+1-j}\left(\begin{array}{c}n \\ j\end{array}\right)$. Then, summing over $j$ in $\mathbb{Z}$ yields that the operator $S_{n}-2$ annihilates the sum $\sum_{j=0}^{n}\left(\begin{array}{l}n \\ j\end{array}\right)$. This method of creative telescoping will be described in $\S 2.1$. Solving the corresponding recurrence, we deduce that $\sum_{j=0}^{n}\left(\begin{array}{c}n \\ j\end{array}\right)=2^{n}$. More generally, the algorithms described in this tutorial input and output linear systems. Typically, the input is a partial differential or recurrence system, while the output is an ordinary differential or recurrence equation, whose resolution is left as a post-processing.

As another example, the Appell $F_{4}$ bivariate hypergeometric function

$$
F_{4}(a, b, c, d ; x, y)=\sum_{m, n=0}^{\infty} \frac{(a)_{m+n}(b)_{m+n}}{m ! n !(c)_{m}(d)_{n}} x^{m} y^{n}
$$

where $(x)_{n}$ denotes the Pochhammer symbol $\Gamma(x+n) / \Gamma(x)$, satisfies linear differential equations (Erdélyi 1981). Let $D_{x}$ denote the operator of derivation with respect to $x$ : this operator acts on a function $f$ by $\left(D_{x} \cdot f\right)(x)=f^{\prime}(x)$. Denoting derivations with respect to other variables in analogously, the Appell $F_{4}$ function is annihilated by

$$
\left\{\begin{array}{l}
x D_{x}\left(x D_{x}+c-1\right)-x\left(x D_{x}+y D_{y}+a\right)\left(x D_{x}+y D_{y}+b\right), \\
y D_{y}\left(y D_{y}+d-1\right)-y\left(x D_{x}+y D_{y}+a\right)\left(x D_{x}+y D_{y}+b\right)
\end{array}\right.
$$


with a similar identification for rational functions in $(x, y)$ as in the previous example. Here, determining an operator which does not involve $y$, i.e., in $x$, $D_{x}$ and $D_{y}$ only, is enough to obtain an ordinary differential equation satisfied by an integral of the Appell function.

More generally, the operator approach applies to mixed difference-differential systems. For example, the Jacobi orthogonal polynomials $P_{n}^{(a, b)}(z)$, viewed as a function $P$ in $(n, a, b, z)$, is annihilated by the classical linear differencedifferential system (Erdélyi 1981)

$$
\left\{\begin{array}{l}
\left(1-z^{2}\right) D_{z}^{2}+(b-a-(a+b+2) z) D_{z}+n(n+a+b+1), \\
(2 n+a+b+2)\left(1-z^{2}\right) S_{n} D_{z} \\
\quad-(n+1)(a-b-(2 n+a+b+2) z) S_{n} \\
-2(n+a+1)(n+b+1), \\
2(n+2)(n+a+b+2)(2 n+a+b+2) S_{n}^{2} \\
-(2 n+a+b+3) \\
\quad\left[(2 n+a+b+2)(2 n+a+b+4) z+\left(a^{2}-b^{2}\right)\right] S_{n} \\
\quad+2(n+a+1)(n+b+1)(2 n+a+b+4), \\
(2 n+a+b+2)(1-z) S_{a}-2(n+a+1)+2(n+1) S_{n}, \\
(2 n+a+b+2)(1+z) S_{b}-2(n+b+1)-2(n+1) S_{n} .
\end{array}\right.
$$

Again, deriving an operator that does not involve $z$ suffices to perform integrations of $P$.

An abstract framework for linear operators is given by Ore algebras, which provide a unifying viewpoint on shift and derivation operators (Chyzak and Salvy 1996). They also provide a polynomial representation for the operators under consideration, generalizing the polynomial representation of the systems (0.1), (0.3) and (0.4). It turns out that the theory of Gröbner bases developed for commutative algebras of polynomials extends to this non-commutative setting. Early work in this area is due to Galligo (1985) in the differential case. Takayama (1989) used an analogous technique for differencedifferential algebras. A general setting was introduced by Kandri-Rody and Weispfenning (1990) and Kredel (1993), and later adapted to Ore algebras (Chyzak and Salvy 1996). In this tutorial, we illustrate applications of noncommutative Gröbner bases to symbolic summation and integration. Only simple facts of the theory of commutative Gröbner bases will be required, for which we refer to (Buchberger 1998) and (Cox et al. 1992).

Gröbner bases are used for various purposes in the context of the symbolic manipulation of linear operators. First, by providing normal forms modulo an ideal, they are crucial to algorithms which compute in finite-dimensional quotient rings. This applies in particular to the algorithm for indefinite summation and integration which we present in Section 1, after setting up the algebraic framework of Ore algebras and recalling how Gröbner bases can be computed there. Next, the use of Gröbner bases for elimination purposes is the keystone of a general method of definite summation and integration called 
creative telescoping. An algorithm is presented in Section 2, together with an extension to multiple summations and integrations. A more efficient version in the case of natural boundaries is obtained by appealing to Gröbner bases for modules. We end the section by applying the algorithm of Section 1 to the definite case. In Section 3, we turn again to the use of Gröbner bases for computing normal forms and obtain algorithms for various other operations. We also present a second method for the indefinite case. Finally, Gröbner bases allow the calculation of ideal dimensions. This concept is related to the theory of holonomy, which we allude to in Section 4, and is used to explain the success of the various methods and to decide the termination of the corresponding algorithms.

\section{Indefinite Summation and Integration}

The algorithms which we illustrate simultaneously apply to summation and integration. Indeed, they share an interpretation in terms of Ore operators which generalize both derivation and difference operators. This algorithmically important fact stems from the essential property of Ore operators to satisfy a skew Leibnitz rule (see (1.1) below) which encompasses both the classical Leibnitz rule for derivations,

$$
(f g)^{\prime}(x)=f(x) g^{\prime}(x)+f^{\prime}(x) g(x)
$$

and the skew Leibnitz rule for the finite difference operator $\Delta$,

$$
\Delta(f g)(x)=f(x+1) \Delta g(x)+(\Delta f(x)) g(x),
$$

where $\Delta f(x)=f(x+1)-f(x)$. Furthermore, Ore operators have a representation in special algebras, namely Ore algebras, which can be regarded as algebras of skew polynomials. Due to the nice properties of these polynomials, a theory of Gröbner bases is available in Ore algebras. To end the section, we present an algorithm for the indefinite case, which will be used for the definite case in $\S 2.4$.

\subsection{Ore Operators, Ore Algebras, Annihilating Ideals}

The purpose of this section is to provide a representation of linear operators which is suitable for symbolic manipulations, and in particular for Gröbner basis calculations. This encoding is in terms of skew polynomials, which encapsulate the difference and differential cases in a single algebraic framework (Cohn 1971). Considering the polynomial form of the operators in (0.1), (0.3) and (0.4), we introduce a generic indeterminate $\partial$ which may either represent a derivation operator, a shift operator, or more general Ore operators (Bronstein and Petkovšek 1994; Ore 1933). This $\partial$ inherits the action on functions 
of the original operator. Introducing several indeterminates $\partial_{i}$, we then describe multivariate cases. Imposing commutation on the $\partial_{i}$ 's, we are led to the definition of Ore algebras (Chyzak and Salvy 1996).

In view of the similarity between the treatments of functions and sequences, we henceforth indifferently use the word "function" for functions and sequences, and more generally for any object on which we apply linear operators. Let $\mathcal{F}$ be an algebra of functions over a commutative field $\mathbb{K}$ and denote the algebra of $\mathbb{K}$-linear endomorphisms of $\mathcal{F}$ by $\operatorname{End}_{\mathbb{K}} \mathcal{F}$.

By analogy with the prime notation for derivations, we denote by $f^{\eta}$ the action of $\eta \in \operatorname{End}_{\mathbb{K}} \mathcal{F}$ on $f \in \mathcal{F}$. An endomorphism $\delta \in \operatorname{End}_{\mathbb{K}} \mathcal{F}$ which satisfies the Leibnitz law $(f g)^{\delta}=f g^{\delta}+f^{\delta} g$ for $f, g \in \mathcal{F}$ is called a derivation or derivation operator. On the other hand, an algebra endomorphism $\sigma \in \operatorname{End}_{\mathbb{K}} \mathcal{F}$ is called a difference or difference operator. Each difference $\sigma$ induces an operator $\theta=\sigma-1$ which satisfies the skew Leibnitz law $(f g)^{\theta}=f^{\sigma} g^{\theta}+f^{\theta} g$ for $f, g \in \mathcal{F}$. More generally, we are interested in pairs $(\sigma, \delta)$ of operators which satisfy such a skew Leibnitz law. Given a difference $\sigma$ on $\mathcal{F}$, an endomorphism $\delta \in \operatorname{End}_{\mathbb{K}} \mathcal{F}$ is called an Ore operator, or a $\sigma$-derivation (Bronstein and Petkovšek 1994; Ore 1933), when

$$
(f g)^{\delta}=f^{\sigma} g^{\delta}+f^{\delta} g \quad \text { for all } f, g \in \mathcal{F} .
$$

This includes classical derivations $\eta$, in which case one considers the pair $(1, \eta)$ where 1 denotes the identity.

We now proceed to describe a representation of subalgebras of $\operatorname{End}_{\mathbb{K}} \mathcal{F}$ generated by $\sigma$-derivations as skew polynomial rings. In order to allow linear operators over various domains of coefficients, we introduce a $\mathbb{K}$-algebra $\mathbb{A}$. In practice, this will always be a polynomial $\operatorname{ring} \mathbb{A}=\mathbb{K}[\mathbf{x}]$ or a rational function field $\mathbb{A}=\mathbb{K}(\mathbf{x})$. In addition, we require $\mathcal{F}$ to include $\mathbb{A}$, so that $\mathbb{A}$ can also be viewed as a subalgebra of $\operatorname{End}_{\mathbb{K}} \mathcal{F}$ by the identification of $a \in \mathbb{A}$ with the operator of multiplication by $a$. In view of the operators that appear in the applications, we distinguish between the general case of a pair $(\sigma, \delta)$ and the special case $(\sigma, \theta)$ for $\theta=\sigma-1$. In the general case, we put the emphasis on the $\sigma$-derivation $\delta$, and a polynomial representation is obtained by encoding $\delta$ through $\partial \cdot f=f^{\delta}$ for $f \in \mathcal{F}$. When additionally $\sigma$ and $\delta$ restrict to a difference and a $\sigma$-derivation on $\mathbb{A}$, the skew polynomial ring $\mathbb{S}=\mathbb{A}[\partial ; \sigma, \delta]$ is defined as the set of polynomials in $\partial$ with coefficients in $\mathbb{A}$, with usual addition and a product defined by associativity from the commutation rule

$$
\partial a=a^{\sigma} \partial+a^{\delta} \quad \text { for } a \in \mathbb{A}
$$

(Cohn 1971). This is the quotient of the free associative algebra in $\partial$ modulo the above relations. In the special case, the emphasis is put on the difference $\sigma=\theta+1$ through the action $\partial \cdot f=f^{\sigma}$ for $f \in \mathcal{F}$. We then consider the skew polynomial ring $\mathbb{A}[\partial ; \sigma, 0]$ governed by the commutation

$$
\partial a=a^{\sigma} \partial \quad \text { for } a \in \mathbb{A} \text {. }
$$


We turn to the multivariate case. Given several pairs of operators $\left(\sigma_{i}, \delta_{i}\right)$ on the same algebra $\mathcal{F}$ with the property that $\sigma_{i}$ and $\delta_{j}$ commute for $i \neq j$, we introduce indeterminates $\partial_{i}$ 's so as to consider the quotient of the free associative algebra in the $\partial_{i}$ 's modulo the relations $\partial_{i} a=a^{\sigma_{i}} \partial_{i}+a^{\delta_{i}}$ for $a \in \mathbb{A}$ and $i=1, \ldots, r$, and $\partial_{i} \partial_{j}=\partial_{j} \partial_{i}$ for $i, j=1, \ldots, r$. Such a skew polynomial ring is called an Ore algebra, denoted $\mathbb{A}[\boldsymbol{\partial} ; \boldsymbol{\sigma}, \boldsymbol{\delta}]$. For convenience, we often denote an Ore algebra $\mathbb{A}[\boldsymbol{\partial} ; \boldsymbol{\sigma}, \boldsymbol{\delta}]$ by the abusive iterated notation $\mathbb{A}\left[\partial_{1} ; \sigma_{1}, \delta_{1}\right] \ldots\left[\partial_{r} ; \sigma_{r}, \delta_{r}\right]$, which could be formalized. When $\mathbb{A}$ is of the form $\mathbb{K}[\mathbf{x}]$, we obtain the special case of polynomial Ore algebras $\mathbb{K}[\mathbf{x}][\boldsymbol{\partial} ; \boldsymbol{\sigma}, \boldsymbol{\delta}]$. Consider the example of the $\mathbb{C}$-algebra $\mathcal{F}=\mathbb{C}(n, a, b)((z))$ of formal Laurent series. The operators (0.4) which annihilate the Jacobi polynomials can be considered in the Ore algebra

$$
\mathbb{O}_{\mathrm{r}}=\mathbb{A}\left[S_{n} ; S_{n}, 0\right]\left[S_{a} ; S_{a}, 0\right]\left[S_{b} ; S_{b}, 0\right]\left[D_{z} ; 1, D_{z}\right] \quad \text { for } \quad \mathbb{A}=\mathbb{C}(n, a, b, z),
$$

but might as well be considered in the polynomial Ore algebra $\mathbb{O}_{\mathrm{p}}$ obtained when $\mathbb{A}=\mathbb{C}[n, a, b, z]$. This distinction will be crucial to treatments by Gröbner bases: the elimination of the indeterminates $n, a, b$ or $z$ is amenable to Gröbner basis methods in $\mathbb{O}_{\mathrm{p}}$ but not in $\mathbb{O}_{\mathrm{r}}$.

For a module $\mathcal{F}$ of functions over an Ore algebra $\mathbb{O}$ and a function $f \in \mathcal{F}$, the set of operators that annihilate the function $f$ has the algebraic structure of a left ideal. It is classically called the annihilating ideal of $f$ and is denoted $A_{\mathbb{O}} f$. This algebraic structure is the basis for the application of Gröbner basis methods. Alternatively, the set $\mathbb{O} \cdot f$ obtained by the action of the Ore algebra $\mathbb{O}$ on the function $f$ is a left $\mathbb{O}$-module, in fact, a left submodule of $\mathcal{F}$. The structure of the module $\mathbb{O} \cdot f$ keeps track of the dependencies between the derivatives $\partial^{\alpha} \cdot f$ of $f$. More specifically, $\mathbb{O} \cdot f \simeq \mathbb{O} / \operatorname{Ann}_{\mathbb{O}} f$.

\subsection{Gröbner Bases in Ore Algebras}

Due to the encoding of Ore operators as skew polynomials, the theory of Gröbner bases extends to Ore algebras. Under mild conditions, the leading term of a product of skew polynomials is the product of the leading terms of the factors. This nice feature avoids any problem of non-noetherianity. The main result originates in works by Kandri-Rody and Weispfenning (1990) and Kredel (1993), which were adapted to Ore algebras by Chyzak and Salvy (1996). It states that polynomial Ore algebras $\mathbb{O}=\mathbb{K}[\mathbf{x}][\boldsymbol{\partial} ; \boldsymbol{\sigma}, \boldsymbol{\delta}]$ such that $\boldsymbol{\partial}$, $\boldsymbol{\sigma}, \boldsymbol{\delta}$ and $\mathbf{x}$ satisfy relations of the type

$$
\partial_{i} x_{j}=\left(a_{i, j} x_{j}+b_{i, j}\right) \partial_{i}+c_{i, j}(\mathbf{x}), \quad 1 \leq i \leq r, \quad 1 \leq j \leq s,
$$

with $b_{i, j} \in \mathbb{K}, a_{i, j} \in \mathbb{K} \backslash\{0\}$ and $c_{i, j} \in \mathbb{K}[\mathbf{x}]$, are left noetherian and that a noncommutative version of Buchberger's algorithm terminates for term orders with respect to which all the $\partial_{i}$ 's are larger than the $x_{i}$ 's. When additionally all the $c_{i, j}$ 's are of total degree at most 1 in $\mathbf{x}$, Buchberger's algorithm terminates 
for any term order on $\mathbf{x}$ and $\boldsymbol{\partial}$. In all cases of termination, Buchberger's algorithm computes a Gröbner basis with respect to the term order. Moreover, the main tools of computational commutative algebra extend to the skew setting as well, and calculations of Hilbert dimensions, polynomials and series, and of Gröbner bases for modules are also available in Ore algebras.

\section{Contiguity Relations for the Appell $F_{4}$ Bivariate Hypergeometric Function}

As an example, we borrow from Takayama (1989) the calculation of contiguity relations for the Appell $F_{4}$ bivariate hypergeometric function defined by Eq. (0.2). The method can be used to derive expressions for the shift and reverse shifts of $F_{4}(a, b, c, d ; x, y)$ with respect to its parameters $a, b, c$ and $d$ in terms of its derivatives with respect to the variables $x$ and $y$. Here, we get a differential expression for $F_{4}(a-1, b, c, d ; x, y)$ which was first obtained by Takayama.

Consider the action of the Ore algebra $\mathbb{O}=\mathbb{K}(x, y)\left[D_{x} ; 1, D_{x}\right]\left[D_{y} ; 1, D_{y}\right]$, where $\mathbb{K}=\mathbb{Q}(a, b, c, d)$, on the algebra $\mathcal{F}=\mathbb{K}((x, y))$ of Laurent power series. The Appell function is annihilated by the operators of (0.3). In fact, $\mathfrak{I}=$ $\mathrm{Ann}_{\mathbb{O}} F_{4}$ is precisely the ideal spanned by these operators. Since

$$
\frac{(a+1)_{m+n}}{(a)_{m+n}}=\frac{(m+n+a)}{a}
$$

the operator $H_{a}=a^{-1}\left(x D_{x}+y D_{y}+a\right)$ satisfies

$$
\left(H_{a} \cdot F_{4}\right)(a, b, c, d ; x, y)=F_{4}(a+1, b, c, d ; x, y)
$$

for this reason, $H_{a}$ is called a step-up operator. We proceed to compute a step-down operator $B_{a}$, i.e., an operator such that $B_{a+1} H_{a}=B_{a} H_{a-1}=1$.

Computing a Gröbner basis for the ideal $\mathfrak{I}$ spanned by the operators (0.3) in $\mathbb{O}$ with respect to a total degree order on $D_{x}$ and $D_{y}$ such that $D_{x} \succ D_{y}$, we obtain

$$
\left\{\begin{array}{l}
2 x y D_{x} D_{y}+\left(x y+y^{2}-y\right) D_{y}^{2} \\
\quad+(a+b-c+1) x D_{x}+(d x+(a+b+1) y-d) D_{y}+a b \\
x D_{x}^{2}-y D_{y}^{2}+c D_{x}-d D_{y} \\
\left(2 x^{2} y^{2}-4 x y^{3}+2 y^{4}-4 x y^{2}-4 y^{3}+2 y^{2}\right) D_{y}^{3} \\
\quad+A_{0,2} D_{y}^{2}+A_{1,0} D_{x}+A_{0,1} D_{y}+A_{0,0}
\end{array}\right.
$$

where each $A_{i, j}$ is a large polynomial in $x$ and $y$. It follows that the quotient ring $\mathbb{O} / \mathfrak{I}$ is a $\mathbb{Q}(a, b, c, d, x, y)$-vector space of dimension 4 which admits $\left\{1, D_{x}, D_{y}, D_{y}^{2}\right\}$ as a basis.

Thus, we set $B_{a+1}=c_{0}+c_{1} D_{x}+c_{2} D_{y}+c_{3} D_{y}^{2}$ with unknowns $c_{i}$ 's. Reducing $B_{a+1} H_{a}-1$ by the Gröbner basis (1.4) and identifying the coefficients 
of $1, D_{x}, D_{y}$ and $D_{y}^{2}$ to zero yields a linear system in $\left(c_{0}, c_{1}, c_{2}, c_{3}\right)$ which we easily solve to obtain the step-down operator.

Note that in the previous calculation, $\mathfrak{I}$ is not required to be $\mathrm{Ann}_{\mathbb{O}} f$, but only a subideal of it such that $\mathbb{O} / \mathfrak{I}$ be finite-dimensional. In the present case, one could prove that $\mathfrak{I}=\mathrm{Ann}_{\mathbb{O}} f$. The problem of working with a subideal of $\mathrm{Ann}_{\mathbb{O}} f$ is that the returned step-down operator could be of higher order.

We refer to (Sturmfels and Takayama 1998) for further results relating to multivariate hypergeometric series.

\section{$1.3 \quad \partial$-Finite Functions}

Many calculations described in this tutorial rely on a property of finiteness satisfied by the input of the algorithms. Informally, the requirement is that the calculations performed by the algorithms take place in a finite-dimensional vector space.

For instance, the key fact that makes the calculations on the example of the Appell function possible is that the quotient $\mathbb{O} / \mathfrak{I}$ is finite-dimensional. Such a situation is rather common. Consider for instance the Jacobi polynomials $P_{n}^{(a, b)}(z)$ regarded as a function $P \in \mathbb{F}((z))$ for $\mathbb{F}=\mathbb{C}(n, a, b)$ and the Ore algebra $\mathbb{O}_{\mathrm{r}}$ defined by Eq. (1.2). It is obvious from (0.4) that the $\mathbb{F}(z)$-vector space $\mathbb{O}_{\mathrm{r}} \cdot P$ is finite-dimensional, and is generated by $\left\{1, S_{n}, D_{z}\right\}$. In fact, computing a Gröbner basis for the set of operators (0.4) with respect to a total degree order on $S_{n}, S_{a}, S_{b}$ and $D_{z}$ such that $D_{z} \prec S_{b} \prec S_{a} \prec S_{n}$, we get:

$$
\left\{\begin{array}{l}
(n+a+b+1) S_{b}-(z-1) D_{z}-(n+a+b+1) \\
(n+a+b+1) S_{a}-(z+1) D_{z}-(n+a+b+1) \\
2(n+1)(n+a+b+1) S_{n}-\left(z^{2}-1\right)(2 n+a+b+2) D_{z} \\
\quad-(n+a+b+1)((2 n+a+b+2) z+a-b) \\
\left(z^{2}-1\right) D_{z}^{2}+((a+b+2) z+a-b) D_{z}-n(n+a+b+1) .
\end{array}\right.
$$

This proves that $\mathbb{O}_{\mathrm{r}} \cdot P=\mathbb{F}(z) P+\mathbb{F}(z)\left(D_{z} \cdot P\right)$ is a vector space of dimension at most 2. From the theory of orthogonal polynomials (Erdélyi 1981), we know that $P$ and $D_{z} \cdot P$ cannot satisfy a linear dependency with rational function coefficients, so that the sum is indeed direct, $\mathbb{O}_{\mathrm{r}} \cdot P$ has dimension 2 and $\mathrm{Ann}_{\mathbb{O}_{\mathrm{r}}} P$ is spanned by the operators (0.4).

On the other hand, the function $f=e^{\sin z} \in \mathcal{F}$ is such that the module $\mathbb{O} \cdot f$ over $\mathbb{O}=\mathbb{C}(z)\left[D_{z} ; 1, D_{z}\right]$ is an infinite-dimensional $\mathbb{C}(z)$-vector space that admits $\left\{(\cos z)^{k} f\right\}_{k \geq 0}$ as a basis. No algorithm of this tutorial applies to functions like $f$.

Formalizing, a function $f \in \mathcal{F}$ is called $\partial$-finite with respect to an Ore algebra $\mathbb{O}=\mathbb{F}[\boldsymbol{\partial} ; \boldsymbol{\sigma}, \boldsymbol{\delta}]$ over a field $\mathbb{F}$ when the vector space $\mathbb{O} \cdot f \simeq \mathbb{O} / \operatorname{Ann}_{\mathbb{O}} f$ is finite-dimensional (Chyzak and Salvy 1996). In the phrasing " $\partial$-finite", $\partial$ is 
merely a generic symbol and bears no relation to the actual $\partial_{i}$ 's that appear in $\mathbb{O}$.

\subsection{Indefinite Summation and Integration}

We present an algorithm which was introduced in (Chyzak 1997) to compute indefinite sums and integrals of $\partial$-finite functions. More generally, the algorithm searches for particular solutions of certain integro-differential problems. The technique is restricted to single summations and integrations, but provides a fast algorithm in the definite case. This will be described in $\S 2.4$.

Consider functions in $\left(x_{0}, \mathbf{x}\right)$; let $\mathbb{O}=\mathbb{K}\left(x_{0}, \mathbf{x}\right)\left[\partial_{0} ; \sigma_{0}, \delta_{0}\right][\boldsymbol{\partial} ; \boldsymbol{\sigma}, \boldsymbol{\delta}]$ be an Ore algebra and $f$ be a $\partial$-finite function with respect to this algebra. Given operators $P \in \mathbb{O}$ and $\Xi \in \mathbb{K}\left(x_{0}\right)\left[\partial_{0} ; \sigma_{0}, \delta_{0}\right]$, we look for a function $F \in \mathbb{O} \cdot f$, if one exists, such that

$$
\Xi\left(x_{0}, \partial_{0}\right) \cdot F=P\left(x_{0}, \mathbf{x}, \partial_{0}, \boldsymbol{\partial}\right) \cdot f .
$$

The key idea of the algorithm is to write $F$ in the form $Q \cdot f$, and then to solve for $Q \in \mathbb{O}$. A solution $F$ found in this way is a $\partial$-finite particular solution of Eq. (1.5). In general, no such solution exists. In this case, the algorithm returns a proof that no solution exists in $\mathbb{O} \cdot f$.

As a special case, when $\partial_{0}$ is a derivation operator and $\Xi=\partial_{0}$, we get an algorithm for indefinite integration. When $\partial_{0}$ is a shift operator and $\Xi=$ $\partial_{0}-1$, we get an algorithm for indefinite summation. In both cases, either we set $P=1$ to integrate or sum the function $f$ itself, or the algorithm applies to any element of the module $\mathbb{O} \cdot f$. When $\Xi=\partial_{0}-1, r=0, \partial_{0}=S_{n}$, $\mathbb{O}=\mathbb{Q}(n)\left[S_{n} ; S_{n}, 0\right]$, and $\mathbb{O} \cdot f$ is of dimension 1 , we get an algorithm for the indefinite summation of hypergeometric terms, that is based on Abramov's classical algorithm (Abramov 1989a, 1989b, 1995; Abramov and Kvashenko 1991; Abramov et al. 1995); this algorithm is an alternative to Gosper's algorithm (Gosper 1978).

\section{Harmonic Summation}

Harmonic summation identities like the indefinite summation

$$
\sum_{k=1}^{n}\left(\begin{array}{l}
k \\
m
\end{array}\right) H_{k}=\left(\begin{array}{c}
n+1 \\
m+1
\end{array}\right)\left(H_{n+1}-\frac{1}{m+1}\right)
$$

are classically proved by summation by parts or by techniques of generating functions. Here, we prove the above identity following the operator approach.

Introducing $f_{n}=\left(\begin{array}{c}n \\ m\end{array}\right) H_{n}$, we show the equivalent form

$$
\sum_{k=1}^{n} f_{k}=\frac{(n+1)^{2}}{(m+1)^{2}} f_{n}-\frac{(n-m)(n-m+1)}{(m+1)^{2}} f_{n+1} .
$$


First, $f$ satisfies the linear recurrence

$$
\begin{array}{r}
(n-m+1)(n-m+2) f_{n+2}-(2 n+3)(n-m+1) f_{n+1} \\
+(n+1)^{2} f_{n}=0
\end{array}
$$

which is obtained by homogenizing the relation

$$
(n+1-m) f_{n+1}=(n+1) f_{n}+1
$$

allowing rational function coefficients in $\mathbb{Q}(n, m)$ only. In addition, one could prove that $f$ cannot satisfy any such homogeneous relation of lower order. It follows that the sequence $f$ is a $\partial$-finite function with respect to the Ore algebra $\mathbb{O}=\mathbb{Q}(n, m)\left[S_{n} ; S_{n}, 0\right]$. We look for an indefinite sum $F$ in the module $\mathbb{O} \cdot f$. Since the latter is a two-dimensional vector space with basis $\left\{f, S_{n} \cdot f\right\}$, we introduce a generic operator $Q=\alpha_{n}+\beta_{n} S_{n}$ such that $F=$ $Q \cdot f$. We impose $\left(S_{n}-1\right) \cdot F=f$, so that we compute $Z=\left(S_{n}-1\right) Q-1$. Our goal is to find rational functions $\alpha$ and $\beta$ such that $Z$ is zero. We have

$$
Z=\beta_{n+1} S_{n}^{2}+\left(\alpha_{n+1}-\beta_{n}\right) S_{n}-\left(\alpha_{n}+1\right)
$$

which we reduce using Eq. (1.7). We obtain

$$
\begin{aligned}
Z= & \left(\alpha_{n+1}+\frac{(2 n+3) \beta_{n+1}}{n-m+2}-\beta_{n}\right) S_{n} \\
& -\left(\alpha_{n}+\frac{(n+1)^{2} \beta_{n+1}}{(n-m+1)(n-m+2)}+1\right) .
\end{aligned}
$$

Setting both terms in parentheses to zero, next uncoupling the recurrence system so as to get rid of $\alpha$, yields the recurrence equation

$$
\begin{aligned}
& (n+2)^{2} \beta_{n+2}-(2 n+3)(n-m+3) \beta_{n+1} \\
& \quad+(n-m+2)(n-m+3) \beta_{n}+(n-m+3)(n-m+2)=0,
\end{aligned}
$$

which is solved for rational solutions by Abramov's algorithm (Abramov 1989a, 1989b, 1995; Abramov et al. 1995). In general, the uncoupling step can be performed using specialized algorithms (Abramov and Zima 1996; Barkatou 1993; Bronstein and Petkovšek 1996), or by a Gröbner basis calculation (see below). Substituting in the system and eliminating $\alpha_{n+1}$ between both equations yields an expression for $\alpha_{n}$. The expressions found are

$$
\alpha_{n}=\frac{(n+1)^{2}}{(m+1)^{2}} \quad \text { and } \quad \beta_{n}=-\frac{(n-m)(n-m+1)}{(m+1)^{2}}
$$

which proves Eq. (1.6). 


\section{Particular Solutions}

The classical method of variation of the constant to search for a particular solution of a non-homogeneous ordinary differential equation returns an output which involves an indefinite integral and a division. On the other hand, the method of the present section searches for explicit linear expressions in the class of $\partial$-finite functions.

As an example, let $\mathbb{O}$ be the Ore algebra $\mathbb{Q}(q, x)\left[D_{x} ; 1, D_{x}\right]$ and consider the equation:

$$
\left(D_{x}^{2}+\frac{1}{x} D_{x}-q^{2}\right) \cdot F(x)=\left(1-x^{2}\right) J_{0}(q x)-2 q^{2} x J_{1}(q x)
$$

where $J_{\nu}$ is the Bessel function of the first kind and order $\nu$ (Erdélyi 1981). This corresponds to the general setting for

$$
f(x)=J_{0}(q x), \quad P=\left(1-x^{2}\right)-2 q x D_{x} \quad \text { and } \quad \Xi=D_{x}^{2}+x^{-1} D_{x}-q^{2} .
$$

Once again $\mathbb{O} \cdot f$ has dimension 2. Introducing $Q=\alpha(x)+\beta(x) D_{x}$ and reducing $Z=\Xi Q-P$ yields a linear differential system, which, once uncoupled, is solved by another algorithm of Abramov's (Abramov 1989a, 1989b; Abramov and Kvashenko 1991). The solution is

$$
\alpha(x)=\frac{2 q-1+x^{2}}{2 q^{2}} \quad \text { and } \quad \beta(x)=\frac{\left(1-q^{3}\right) x}{q^{2}} .
$$

Denoting by $I_{\nu}$ and $K_{\nu}$ the modified Bessel functions of the first and second kind and of order $\nu$ (Erdélyi 1981), the general solution to the differential equation above is:

$$
\frac{2 q-1+x^{2}}{2 q^{2}} J_{0}(q x)+\frac{\left(q^{3}-1\right) x}{q^{3}} J_{1}(q x)+C_{1} I_{0}(q x)+C_{2} K_{0}(q x),
$$

since both $I_{0}(q x)$ and $K_{0}(q x)$ are annihilated by $\Xi$.

\section{Multivariate Extension}

In other cases, we do not deal with a function $f$ in a single variable $x_{0}$, but in variables $\left(x_{0}, \mathbf{x}\right)$. We then consider the annihilating ideal of $f$ with respect to an Ore algebra $\mathbb{O}=\mathbb{K}\left(x_{0}, \mathbf{x}\right)\left[\partial_{0} ; \sigma_{0}, \delta_{0}\right][\boldsymbol{\partial} ; \boldsymbol{\sigma}, \boldsymbol{\delta}]$. Gröbner bases are then used for several purposes:

1. a Gröbner basis of $\mathfrak{I}=\operatorname{Ann}_{\mathbb{O}} f$ yields a basis of the $\mathbb{K}$-vector space $\mathbb{O} / \mathfrak{I}$ and determines how many undetermined coefficients have to be introduced in the generic operator $Q$;

2. the reduction of $\Xi Q-P$ is then performed using the same Gröbner basis; 
3. the uncoupling step can be performed by computing the Gröbner basis of an $\mathbb{O}$-module.

Let us detail the last point. Assume that $N$ undetermined coefficients $\eta_{i}$, for $i=1, \ldots N$, have been introduced in $Q$; in other words, we assume that $\mathbb{O}$. $f$ has dimension $N$. We then introduce the free module $\mathfrak{M}=\bigoplus_{i=0}^{N} \mathbb{O} \cdot e_{i}$ over new indeterminates $e_{i}$ and interpret any linear expression in the $\eta_{i}$ 's and their derivatives as an element of $\mathfrak{M}$ by mapping $\eta_{i}$ onto $e_{i}$ and making the nonhomogeneous terms act on $e_{0}$. For instance, in the example of the harmonic summation above, we use $e_{1}$ and $e_{2}$ to keep track of the operators that act on $\alpha$ and $\beta$, respectively. An expression $U \cdot \alpha+V \beta+r$ for $U, V \in \mathbb{O}$ and $r \in \mathbb{Q}(n, m)$ is then encoded by $r \cdot e_{0}+U \cdot e_{1}+V \cdot e_{2}$.

In particular, any linear relation between the $\eta_{i}$ 's and their derivatives has its counterpart in the $\mathbb{O}$-module $\mathfrak{M}$. We encode the set of all such equations as the submodule $\mathfrak{N}$ of $\mathfrak{M}$ spanned by the polynomials obtained after encoding the coefficients of the reduced form of $Z=\Xi Q-P$ with respect to $\left(\partial_{0}, \partial\right)$. In order to uncouple the system corresponding to $Z$, we compute a Gröbner basis for $\mathfrak{N}$ with respect to a term order that sorts the $e_{i}$ 's lexicographically. We get a triangularized system, as required. Following up the example of the uncoupling step in the harmonic summation above, the expression $Z$ that is defined by Eq. (1.8) is encoded by

$$
\left\{\begin{array}{l}
(n-m+2) S_{n} \cdot e_{1}+\left[(2 n+3) S_{n}-(n-m+2)\right] \cdot e_{2}, \\
(n-m+1)(n-m+2) \cdot\left(e_{0}+e_{1}\right)+(n+1)^{2} S_{n} \cdot e_{2},
\end{array}\right.
$$

which generates a submodule $\mathfrak{N}$ of $\mathfrak{M}=\bigoplus_{i=0}^{2} \mathbb{O} \cdot e_{i}$. We compute a Gröbner basis for $\mathfrak{N}$ with respect to a term order that satisfies $e_{2} \succ e_{1} \succ e_{0}$. This eliminates $e_{2}$ and we obtain

$$
\begin{array}{r}
{\left[(n+2)^{2} S_{n}^{2}-(2 n+3)(n-m+3) S_{n}+(n-m+2)(n-m+3)\right] \cdot e_{1}} \\
+(n-m+3)(n-m+2) \cdot e_{0}
\end{array}
$$

which represents Eq. (1.9).

\section{Definite Summation and Integration}

A simple algorithm for definite summation and integration is based on a brute-force elimination by a Gröbner basis calculation and appeals to the method of creative telescoping (§2.1). Since several indeterminates can be eliminated simultaneously, this general method applies to multiple summations and integrations as well (§2.2). However, a partial elimination is always preferable. When in addition certain analytical conditions are satisfied, more specifically in the case of so-called natural boundaries, a partial elimination is made possible by the calculation of Gröbner basis for modules ( $(2.3)$. The 
corresponding algorithm also deals with the case of multiple summations and integrations. We finish the section with an algorithm that does not appeal to elimination by Gröbner bases, but is based on the algorithm of $\S 1.4$. It is also faster than the brute-force method, but restricted to single summations and integrations $(\S 2.4)$.

\subsection{Creative Telescoping and Elimination by Gröbner Bases}

In this section, we turn to the problem of definite summation and integration by a method based on elimination (Almkvist and Zeilberger 1990). We exemplify the method by the integration of the function $f$ defined by

$$
f_{n}(x)=\frac{e^{-p x} T_{n}(x)}{\sqrt{1-x^{2}}}, \quad \text { where } \quad T_{n}(x)=\frac{n}{2} \sum_{k=0}^{\lfloor n / 2\rfloor}(-1)^{k} \frac{(n-k-1) !}{k !(n-2 k) !}(2 x)^{n-2 k}
$$

denotes the Chebyshev orthogonal polynomials of the first kind (Erdélyi 1981). More specifically, we proceed to prove (Prudnikov et al. 1986: $§ 2.18 .1$, Eq. (10)),

$$
\int_{-1}^{+1} \frac{e^{-p x} T_{n}(x)}{\sqrt{1-x^{2}}} d x=\pi(-1)^{n} I_{n}(p)
$$

This identity appears for example in the study of the fundamental modes of vibration of a drum membrane in interaction with a mallet (Joly and Rhaouti 1997).

The most basic algorithm works as follows. We view $f$ as a member of $\mathcal{F}=\mathbb{C}(p, n)((x))$ and make the algebra $\mathbb{O}_{\mathrm{r}}=\mathbb{C}(p, n, x)\left[D_{x} ; 1, D_{x}\right]\left[S_{n} ; S_{n}, 0\right]$ act on $\mathcal{F}$. Then, $f$ is annihilated by the special operator

$$
D_{x}\left(S_{n}^{2}-1\right)+p S_{n}^{2}-2(n+1) S_{n}-p,
$$

and this operator suffices to obtain integrals of $f$ with respect to $x$, as explained below. The peculiarity of the previous operator is that it does not involve the variable $x$ of integration in its coefficients. Moreover, one can show within the theory of holonomy that such an eliminated operator exists and can be computed for a large class of functions (Zeilberger 1990b); see also Section 4. A similar phenomenon is exploited for creative telescoping in the summation case, where a special operator that does not involve the index of summation in its coefficients suffices to perform summation (Zeilberger 1991).

Indeed, applying the operator (2.2) to $f$ and integrating between $u$ and $v$ yields the non-homogeneous recurrence

$$
\left[\left(S_{n}^{2}-1\right) \cdot f\right]_{u}^{v}+\left(p S_{n}^{2}-2(n+1) S_{n}-p\right) \cdot \int_{u}^{v} f_{n}(x) d x=0 .
$$


A series expansion indicates that the left term tends to 0 for any $n$ when $x$ goes to \pm 1 , so that for $u=-1$ and $v=+1$, the integral satisfies a homogeneous recurrence. Note that this recurrence could have been obtained directly by setting $D_{x}=0$ in (2.2), if we had predicted the cancellation of the left term in Eq. (2.3) beforehand. Solving the second order recurrence can be done automatically. This yields two $\mathbb{C}$-independent solutions, $(-1)^{n} I_{n}(p)$ and $(-1)^{n} K_{n}(p)$. To complete the proof of Eq. (2.1), one needs to determine which solution of the two-dimensional vector space of solution the integral is. This is achieved by considering initial conditions at $n=0$ and $n=1$.

It remains to explain how an operator like (2.2) can be obtained. First, the polynomials $T_{n}(x)$ are $\partial$-finite; the following operators vanishing at $f$ are variations on classical equations satisfied by $T_{n}(x)$ (Erdélyi 1981), obtained by the change of function $T_{n}(x)=\sqrt{1-x^{2}} e^{p x} f_{n}(x)$ :

$$
\left\{\begin{array}{l}
\left(x^{2}-1\right) D_{x}^{2}+\left(2 p x^{2}+3 x-2 p\right) D_{x}+p^{2} x^{2}+3 p x-n^{2}-p^{2}+1 \\
\left(x^{2}-1\right) D_{x} S_{n}+\left(p x^{2}-n x-p\right) S_{n}+n+1, \quad S_{n}^{2}-2 x S_{n}+1 .
\end{array}\right.
$$

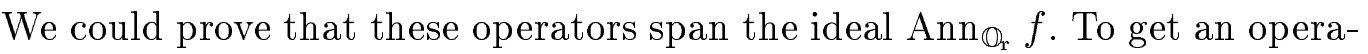
tor that is free from $x$, we would like to compute the intersection of this ideal with the subalgebra $\mathbb{C}(p, n)\left[D_{x}\right]\left[S_{n} ; S_{n}, 0\right]$ of $\mathbb{O}_{\mathrm{r}}$. To this end, it seems natural to perform this elimination by computing a Gröbner basis for a suitable term order. However, such an elimination is tractable by Gröbner basis calculations only if $x$ appears in a polynomial way in the algebra. We therefore compute the Gröbner basis of the ideal spanned by the system (2.4) in the polynomial Ore algebra $\mathbb{O}_{\mathrm{p}}=\mathbb{C}(p)[n, x]\left[D_{x} ; 1, D_{x}\right]\left[S_{n} ; S_{n}, 0\right]$ for a term order on $n, x, D_{x}$ and $S_{n}$ that sets $x$ lexicographically greater than the other indeterminates (i.e., $x \succ n^{i} D_{x}^{j} S_{n}^{k}$ for any $i, j$ and $k$ ) and breaks ties by a total degree order such that $S_{n} \succ D_{x} \succ n$. This yields

$$
\left\{\begin{array}{l}
S_{n}^{2} D_{x}-D_{x}-2 S_{n}-2 n S_{n}+p S_{n}^{2}-p \\
x D_{x}-S_{n} D_{x}+p x-p S_{n}+n+1, \quad 2 x S_{n}-S_{n}^{2}-1
\end{array}\right.
$$

The operator (2.2) is obtained as the only operator which does not involve $x$.

\subsection{Multiple Summations and Integrations}

The previous method by brute-force elimination also applies to multiple summations and integrations. For instance, we compute

$$
\sum_{k=0}^{\infty} \sum_{\ell=0}^{\infty} \frac{(2 k+2 \ell+n+m) !}{(k+n) !(\ell+m) ! k ! \ell !}\left(\frac{x}{4}\right)^{k+\ell}
$$

and prove that this double sum admits the (infinite) single sum representation

$$
\frac{n !}{(n-m) ! m !}{ }_{4} F_{3}\left(\begin{array}{c}
\frac{n+m}{2}+1, \frac{n+m}{2}+1, \frac{n+m+1}{2}, \frac{n+m+1}{2} \\
n+m+1, n+1, m+1
\end{array} \mid 4 x\right),
$$


in terms of the hypergeometric ${ }_{4} F_{3}$ function. The double sum describes the probability of reaching the point $(n, m)$ by a random walk on the integer lattice $\mathbb{N}^{2}$ starting from the origin, with probability $x / 4$ to proceed in any of the four directions and probability $1-x$ to stop the walk at each step (Trigg 1996).

Considering the Ore algebra $\mathbb{K}(k, \ell, x)\left[S_{k} ; S_{k}, 0\right]\left[S_{\ell} ; S_{\ell}, 0\right]\left[D_{x} ; 1, D_{x}\right]$ over the field $\mathbb{K}=\mathbb{Q}(n, m)$, the following system of operators describes the summand $s$ as a $\partial$-finite function:

$$
\left\{\begin{array}{l}
x D_{x}-(k+\ell) \\
4(k+1)(k+1+n) S_{k} \\
\quad-x(2(k+\ell)+n+m+1)(2(k+\ell+1)+n+m) \\
4(\ell+1)(\ell+1+m) S_{\ell} \\
\quad-x(2(k+\ell)+n+m+1)(2(k+\ell+1)+n+m) .
\end{array}\right.
$$

These operators are obtained by computing normal forms for $\left(D_{x} \cdot s\right) / s$, $\left(S_{k} \cdot s\right) / s$ and $\left(S_{\ell} \cdot s\right) / s$ respectively, which turn out to be rational functions in $\mathbb{K}(k, \ell, x)$.

In view of the creative telescoping, we compute a Gröbner basis for the ideal spanned by (2.7) in the polynomial Ore algebra

$$
\mathbb{K}(x)[k, \ell]\left[S_{k} ; S_{k}, 0\right]\left[S_{\ell} ; S_{\ell}, 0\right]\left[D_{x} ; 1, D_{x}\right] .
$$

To perform the elimination, we use a term order on the five polynomial indeterminates of the algebra so that $k$ and $\ell$ are lexicographically greater than $S_{k}, S_{\ell}$ and $D_{x}$. Ties are broken by a total degree order on $k$ and $\ell$ such that $k \prec \ell$ on the one hand, and by a total degree order on $S_{k}, S_{\ell}$ and $D_{x}$ such that $S_{k} \prec S_{\ell} \prec D_{x}$ on the other hand. The Gröbner basis found has the following shape:

$$
\begin{cases}D_{x}^{5} S_{k}^{2} S_{\ell}^{2}+\langle 307 \text { l.o.t. }\rangle, & D_{x}^{4} S_{k}^{3} S_{\ell}^{3}+\langle 511 \text { l.o.t. }\rangle, \\ k+\ell-x D_{x}, & k S_{k}^{2} S_{\ell}^{2}+\langle 192 \text { l.o.t. }\rangle, \\ k S_{k} S_{\ell}+\langle 27 \text { l.o.t. }\rangle, & k D_{x}^{2} S_{k}+\langle 72 \text { l.o.t. }\rangle, \\ k S_{k} S_{\ell}^{3}+\langle 467 \text { l.o.t. }\rangle, & k D_{x}^{2} S_{\ell}^{2}+\langle 103 \text { l.o.t. }\rangle, \\ k^{2} S_{\ell}+\langle 15 \text { l.o.t. }\rangle, & k^{2} S_{k}+\langle 14 \text { l.o.t. }\rangle\end{cases}
$$

where " $p$ l.o.t." stands for " $p$ lower order terms", and where each polynomial has been made monic. Both operators of the first line contain neither $k$ nor $\ell$. It has been noted in the previous section that a recurrence for the integral of Eq. (2.1) is obtained by setting $D_{x}=0$ in the eliminated operator (2.2). In the present case, summing over $k$ and $\ell$ in $(0, \infty)$ corresponds to setting $S_{k}=1$ and $S_{\ell}=1$ in the first two operators of (2.8). This yields two operators of $\mathbb{K}(x)\left[D_{x} ; 1, D_{x}\right]$ of degree 4 and 5 in $D_{x}$, respectively. Computing a Gröbner basis with respect to a term order on $D_{x}$, we get their right gcd, namely the 
following operator of degree 4:

$$
\begin{aligned}
& 4 x^{3}(1-4 x) D_{x}^{4}+8 x^{2}((s+3)-2(2 s+9) x) D_{x}^{3} \\
& \quad+4 x\left(\left(s^{2}+p+6 s+7\right)-\left(6 s^{2}+42 s+77\right) x\right) D_{x}^{2} \\
& \quad+4\left((p+s+1)(s+1)-(2 s+5)\left(s^{2}+5 s+7\right) x\right) D_{x}-(s+1)^{2}(s+2)^{2}
\end{aligned}
$$

for $s=n+m$ and $p=n m$. The previous calculation reduces in fact to a skew Euclidean algorithm (Bronstein and Petkovšek 1996; Chyzak and Salvy 1996; Ore 1933). Solving the corresponding differential equation and taking into account four initial values at 0 yields the hypergeometric series representation of (2.6) for the double sum.

Following an approach that is not based on Gröbner bases, Wegschaider (1997) obtained a fast algorithm tailored for the calculation of binomial multiple sums like (2.5).

\subsection{Takayama's Algorithm and Gröbner Bases of Mod- ules}

We proceed to describe how under certain analytical conditions, the bruteforce elimination of the previous algorithms can be replaced by a moderated elimination.

Consider a function $f$ in $\left(x_{0}, \mathbf{x}\right)$ to be summed or integrated with respect to $x_{0}$, and let $\mathbb{O}_{\mathrm{e}}$ be the Ore algebra $\mathbb{K}(\mathbf{x})\left[x_{0}\right]\left[\partial_{0} ; \sigma_{0}, \delta_{0}\right][\boldsymbol{\partial} ; \boldsymbol{\sigma}, \boldsymbol{\delta}]$ that would be used by the algorithms of the previous sections. The key ingredient of the method is an annihilating operator like (2.2), of the form

$$
\partial_{0} Q\left(\mathbf{x}, \partial_{0}, \boldsymbol{\partial}\right)+P(\mathbf{x}, \boldsymbol{\partial})
$$

where $x_{0}$-the variable of integration when $\partial_{0}=D_{x_{0}}$; the index of summation when $\partial_{0}=S_{x_{0}}-1$-neither appears in $P$ nor in $Q$. It was observed by Almkvist and Zeilberger (1990) that the elimination of $x_{0}$ is more than needed. In fact, an annihilating operator of the form

$$
\partial_{0} Q\left(x_{0}, \mathbf{x}, \partial_{0}, \boldsymbol{\partial}\right)+P(\mathbf{x}, \boldsymbol{\partial})
$$

suffices for the next steps of creative telescoping. On the other hand, the term in $Q$ results in a variational term $[Q \cdot f]_{x_{0}=u}^{x_{0}=v}$, like in Eq. (2.3). Cases where this term can be predicted to evaluate to zero are called summation and integration over natural boundaries. We then only need to compute $P$.

An algorithm to deal with this case in a differential framework is given in (Takayama 1990a, 1990b), and was further elaborated to accommodate difference operators in (Chyzak and Salvy 1996). The key idea is that the 
annihilating operators of the form (2.10) may be multiplied by $\mathbf{x}, \partial_{0}$ and $\boldsymbol{\partial}$, but not by $x_{0}$, and thus constitute a module over the algebra

$$
\mathbb{O}_{\mathrm{m}}=\mathbb{K}(\mathbf{x})\left[\partial_{0} ; \sigma_{0}, \delta_{0}\right][\boldsymbol{\partial} ; \boldsymbol{\sigma}, \boldsymbol{\delta}]
$$

The annihilating ideal $\mathfrak{I}=\operatorname{Ann}_{\mathbb{O}_{\mathrm{e}}} f$ of $\mathbb{O}_{\mathrm{e}}$ is then viewed as an $\mathbb{O}_{\mathrm{m}}$-module. As such, it is of infinite type and is generated by the products of the (finitely many) generators of $\mathfrak{I}$ with the (infinitely many) powers of $x_{0}$. The algorithm then proceeds by truncation of the module with respect to the degree in $x_{0}$ : one computes an elimination Gröbner basis for the $\mathbb{O}_{\mathrm{m}}$-module spanned by all operators up to a given degree in $x_{0}$; if an operator of the type (2.10) is found, the algorithm returns the corresponding $P$; otherwise the maximum degree in $x_{0}$ is increased. The discussion of the termination of this algorithm is based on the concept of holonomy and ideal dimension, and is postponed until Section 4.

The algorithm also generalizes to multiple summations and integrations; in this case, one introduces an algebra $\mathbb{O}_{\mathrm{e}}$ is of the form

$$
\mathbb{K}(\mathbf{x})\left[x_{0}, \ldots, x_{s}\right]\left[\partial_{0} ; \sigma_{0}, \delta_{0}\right] \ldots\left[\partial_{s} ; \sigma_{s}, \delta_{s}\right][\boldsymbol{\partial} ; \boldsymbol{\sigma}, \boldsymbol{\delta}]
$$

to sum or integrate over $x_{0}, \ldots, x_{s}$ simultaneously. The form of the crucial operator (2.10) to look for then becomes

$$
\partial_{0} Q_{0}\left(x_{0}, \mathbf{x}, \partial_{0}, \ldots, \partial_{s}, \boldsymbol{\partial}\right)+\cdots+\partial_{s} Q_{s}\left(x_{s}, \mathbf{x}, \partial_{0}, \ldots, \partial_{s}, \boldsymbol{\partial}\right)+P(\mathbf{x}, \boldsymbol{\partial})
$$

We now proceed to exemplify the algorithm with a double summation.

\section{Gordon's Generalization of the Rogers-Ramanujan Identities}

An extension of the famous Rogers-Ramanujan identities (Rogers 1894) is due to Gordon (1961) and yields multiple summations identities. Gordon's theorem states that the partitions of $n$ of the form $\left(\lambda_{1}, \ldots, \lambda_{s}\right)$ where $\lambda_{i}-$ $\lambda_{i+k-1} \geq 2$ for each $i$ and at most $r-1$ of the $\lambda_{i}$ 's equal 1 are equinumerous to the partitions of $n$ into parts that are not congruent to $0, r$ or $-r$ modulo $2 k+$ 1. We recover the Rogers-Ramanujan identities for $k=r=2$ and $k=$ $r+1=2$. They possess an infinite analytic version due to Andrews (1974). For instance for $(k, r)=(3,3)$, we have

$$
\sum_{j=0}^{\infty} \sum_{i=0}^{\infty} \frac{q^{(i+j)^{2}+j^{2}}}{(q ; q)_{i}(q ; q)_{j}}=\frac{\left(q^{3} ; q^{7}\right)_{\infty}\left(q^{4} ; q^{7}\right)_{\infty}}{(q ; q)_{\infty}}
$$

where $(x ; q)_{n}$ denotes the $q$-Pochhammer symbol $(1-x)(1-q x) \ldots\left(1-q^{n-1} x\right)$. More generally, each instance of the theorem above for a fixed $k$ yields a $k$-fold summation identity which is in principle tractable by the algorithm of this 
section. Moreover, these identities can be obtained as a limiting case of a finite analytic version due to Paule (1985). For $k=r=3$, the identity reads

$$
\sum_{j=0}^{n} \sum_{i=0}^{n-j} \frac{q^{(i+j)^{2}+j^{2}}}{(q ; q)_{n-i-j}(q ; q)_{i}(q ; q)_{j}}=\sum_{k=-n}^{n} \frac{(-1)^{k} q^{7 / 2 k^{2}+1 / 2 k}}{(q ; q)_{n+k}(q ; q)_{n-k}} .
$$

To prove this double summation identity, we consider the algebra $\mathcal{F}$ of sequences from $\mathbb{N}^{3}$ to $\mathbb{Q}((q))$ indexed by $(i, j, n)$ and view the summand $f$ as an element of $\mathcal{F}$. We introduce the shift operators $S_{i}, S_{j}$ and $S_{n}$, together with the operators of multiplication by $q, q^{i}, q^{j}$ and $q^{n}$. Those operators generate the algebra $\mathbb{O}=\mathbb{Q}\left(q, q^{i}, q^{j}, q^{n}\right)\left[S_{i} ; S_{i}, 0\right]\left[S_{j} ; S_{j}, 0\right]\left[S_{n} ; S_{n}, 0\right]$ of $\mathbb{Q}$-linear operators on $\mathcal{F}$. These are known as $q$-calculus operators, and satisfy the commutations

$$
S_{i} q^{i}=q q^{i} S_{i}, \quad S_{j} q^{j}=q q^{j} S_{j}, \quad S_{n} q^{n}=q q^{n} S_{n}
$$

Then, $f$ is a $\partial$-finite function since is vanishes at

$$
\left\{\begin{array}{l}
\left(1-q q^{i}\right) q^{j} S_{i}-q q^{i}\left(q^{i} q^{j}-q^{n}\right), \quad\left(1-q q^{j}\right) q^{i} S_{j}-q q^{j}\left(q^{j} q^{i}-q^{n}\right), \\
\left(q^{i} q^{j}-q q^{n}\right) S_{n}-q^{i} q^{j}
\end{array}\right.
$$

This system is obtained by computing normal forms for the quotients $\frac{f_{i+1, j, n}}{f_{i, j, n}}$, $\frac{f_{i, j+1, n}}{f_{i, j, n}}$ and $\frac{f_{i, j, n+1}}{f_{i, j, n}}$ respectively, which turn out to be rational functions in $q$, $q^{i}, q^{j}$ and $q^{n}$. We perform the algorithm described above. The ideal spanned by (2.13) is precisely $\mathrm{Ann}_{\mathbb{O}} f$ (otherwise $f$ would have to be a constant). We compute the set of products of each operator $p$ in $(2.13)$ by $\left(q^{i}\right)^{\alpha}\left(q^{j}\right)^{\beta}$ for $\alpha+\beta \leq 7-d$, where $d$ is the total degree of $p$ in $q^{i}$ and $q^{j}$. This yields 32 operators.

We then make $S_{i}$ and $S_{j}$ disappear from them. The point is that in the present case, the eliminated operator (2.11) takes the form

$\left(S_{i}-1\right) Q_{0}\left(q^{i}, q^{j}, q^{n}, S_{i}, S_{j}, S_{n}\right)+\left(S_{j}-1\right) Q_{1}\left(q^{i}, q^{j}, q^{n}, S_{i}, S_{j}, S_{n}\right)+P\left(q^{n}, S_{n}\right)$,

where we need not compute the $Q_{i}$ 's, but only $P$. Furthermore, $P$ can be obtained from the previous eliminated operator after two Euclidean divisions on the left by $S_{i}-1$ next by $S_{j}-1$. Performing these divisions before any Gröbner basis calculation, we are led to Gröbner basis calculations in an algebra over two indeterminates less, which speeds up calculations. The divisions are easily performed by following the rule

$$
\left(q^{i}\right)^{\alpha} S_{i}^{\beta}=(-1)^{\beta} q^{-\alpha \beta}\left(q^{i}\right)^{\alpha},
$$

which holds modulo the right ideal spanned by $S_{i}-1$. Next, a Gröbner basis is computed with respect to a term order on $q^{i}, q^{j}$ and $S_{n}$ that eliminates $q^{i}$ and $q^{j}$, but with no multiplication by $q^{i}$ and $q^{j}$ allowed. In fact, this calculation 
takes place in the $\mathbb{Q}(n)\left[S_{n} ; S_{n}, 0\right]$-module with the products $\left(q^{i}\right)^{\alpha}\left(q^{j}\right)^{\beta}$ 's as a basis, and with an ordering on this basis which makes $q^{i}$ and $q^{j}$ disappear. We obtain the third-order operator

$$
\begin{aligned}
& \left(q^{3} q^{n}-1\right) S_{n}^{3} \\
& +\left[q^{10}\left(q^{n}\right)^{4}+q-q^{8}\left(q^{n}\right)^{3}+q^{6}\left(q^{n}\right)^{2}+q^{5}\left(q^{n}\right)^{2}-q^{4} q^{n}-q^{3} q^{n}+q^{2}+1\right] S_{n}^{2} \\
& -q\left(1+q^{5}\left(q^{n}\right)^{2}+q^{4}\left(q^{n}\right)^{2}-q^{3} q^{n}+q^{2}+q\right) S_{n}+q^{3} .
\end{aligned}
$$

Repeating the same process on the right-hand side, or using another summation algorithm (Paule and Riese 1997), we obtain the same operator. We complete the proof of Eq. (2.12) by checking that both sides agree for $n=1$, 2 and 3 .

\subsection{Zeilberger's Fast Algorithm and its $\partial$-Finite Exten- sion}

The algorithm described in $\S 1.4$ for indefinite summation and integration also applies to definite summation and integration via creative telescoping (Chyzak 1997). Consider an Ore algebra $\mathbb{O}_{\mathrm{r}}=\mathbb{K}\left(x_{0}, x_{1}\right)\left[\partial_{0} ; \sigma_{0}, \delta_{0}\right]\left[\partial_{1} ; \sigma_{1}, \delta_{1}\right]$ and let $f$ be a $\partial$-finite function with respect to $\mathbb{O}_{\mathrm{r}}$. The method of creative telescoping relies on the search for a function $P \cdot f$ in the module $\mathbb{O}_{\mathrm{r}} \cdot f$, which admits an indefinite sum or integral $F=Q \cdot f$ in the same module. This search corresponds to the search for an eliminated operator (2.10). In view of the summation or integration with respect to $x_{0}$, the method also requires $P$ to be independent from $\left(x_{0}, \partial_{0}\right)$. Assuming such a $P$ is known, the algorithm for the indefinite case can be used to compute $F=Q \cdot f$. The idea of the algorithm in the definite case is therefore to introduce $P$ in the undetermined form $P=\sum_{i=0}^{L} \eta_{i}\left(x_{1}\right) \partial_{1}^{i}$ and to solve for $Q$ and rational $\eta_{i}$ 's simultaneously. Either a solution is found, or one gets a proof that no solution exists with $P$ of degree $L$, in which case the search is repeated with a higher degree $L$ of $P$. The discussion of the termination of this algorithm is similar to that of the algorithm of Section 2.3, and requires concepts to be introduced in Section 4 .

\section{Calkin's Curious Identity}

Calkin (1994) proved the identity

$$
\sum_{k=0}^{n}\left(\sum_{j=0}^{k}\left(\begin{array}{l}
n \\
j
\end{array}\right)\right)^{3}=\frac{n}{2} 8^{n}+8^{n}-\frac{3 n}{4} 2^{n}\left(\begin{array}{c}
2 n \\
n
\end{array}\right)
$$

by manipulations of summation identities. This identity yields the expected value of the maximum of three independent Bernoulli random variables. Computing a closed form for such a left-hand side is now routine work for a computer. We now sketch the computer proof obtained by the algorithm described above. 
Recall from the introduction that the binomial coefficients are annihilated by (0.1). It turns out that (0.1) already is is a Gröbner basis. Computing the inner indefinite sum by the algorithm of $\S 3.2$ yields the Gröbner basis

$$
(n+1-k) S_{n}+k S_{k}+k-2(n+1), \quad(k+1) S_{k}^{2}-(n+1) S_{k}+n-k
$$

of the annihilating ideal of $\sum_{j=0}^{k}\left(\begin{array}{l}n \\ j\end{array}\right)$. Next, computing the cube of this sum by the algorithm of $\S 3.1$ yields a Gröbner basis of the following form:

$$
S_{n} S_{k}+\langle 76 \text { l.o.t. }\rangle, \quad S_{n}^{2}+\langle 249 \text { l.o.t. }\rangle, \quad S_{k}^{3}+\langle 239 \text { l.o.t. }\rangle
$$

where " $p$ l.o.t." stands for " $p$ lower order terms". We introduce undetermined rational functions $\phi_{i}(k), \psi(k)$ and $\eta_{i}$, each of which depends on $n$, and we set

$$
P=\eta_{0}+\eta_{1} S_{n}+\eta_{2} S_{n}^{2}, \quad \text { and } \quad Q=\phi_{0}(k)+\phi_{1}(k) S_{k}+\phi_{2}(k) S_{k}^{2}+\psi(k) S_{n} .
$$

Reducing $\left(S_{k}-1\right) Q-P$ by the Gröbner basis (2.14) and extracting the coefficients of $1, S_{n}, S_{n}^{2}$ and $S_{k}$ yields a linear system of four first order recurrence equations in the $\phi_{i}$ 's and $\psi$. Uncoupling the system by any method suggested in $§ 1.4$ yields a non-homogeneous linear recurrence of order 4 in $\psi(k)$, in which the $\eta_{i}$ 's appear linearly in the non-homogeneous part. All the coefficients of this equation are (large) polynomials of degree 19 or 20 in $k$ with polynomial coefficients in $n$. We then solve this equation by an extension of Abramov's algorithm (Abramov 1989a, 1989b, 1995). This variant mimics the classical extension of Gosper's algorithm described in (Zeilberger 1991), and corresponds to searching for a rational solution $\psi$ together with values of the $\eta_{i}$ 's which allow such a solution to exist. A solution is given by

$$
\left\{\begin{array}{l}
\eta_{0}=-4(2 n+1), \quad \eta_{1}=-(7 n+12), \quad \eta_{2}=n+1, \quad \psi(k)=\frac{N(k)}{D(k)} \\
D(k)=(k-2 n-1)\left(k^{2}+(1-2 n) k+2\left(n^{2}+n+1\right)\right)(k-n-1)^{3}
\end{array}\right.
$$

where $N(k)$ is a polynomial of degree 7 in $k$. Substituting back into the remaining equations yields values for the $\phi_{i}(k)$ 's, each of which is a rational function with denominator $D(k)$. At this point, summing for $k$ over the integer interval $(0, r)$ yields

$$
\left[\left(Q\left(n, k, S_{n}, S_{k}\right) \cdot h\right)(n, k)\right]_{k=0}^{k=r+1}+\sum_{k=0}^{r}\left(P\left(n, S_{n}\right) \cdot h\right)(n, k)=0
$$

where $h_{n, k}=\left(\sum_{j=0}^{k}\left(\begin{array}{c}n \\ j\end{array}\right)\right)^{3}$, provided that $Q \cdot h$ may be evaluated on $(0, r)$. Actually, the previous equation holds for $r \leq n-3$ only, due to the singularity in $n-k-1$ in the denominator $D$ of $Q$. We thus set $r=n-3$ to get the non-homogeneous recurrence equation

$$
\left(P\left(n, S_{n}\right) \cdot H\right)(n)=\sum_{i=0}^{2} \eta_{i}(n) \sum_{k=n-2}^{n+i} h_{n+i, k}-\left[\left(Q\left(n, k, S_{n}, S_{k}\right) \cdot h\right)(n, k)\right]_{k=0}^{k=n-2}
$$


where $H_{n}$ is the sum $\sum_{k=0}^{n} h_{n, k}$ for which we are looking for a closed form. One easily evaluates the non-homogeneous term since $h_{n, n+i}=\left(2^{n}\right)^{3}$ for $i \geq 0$ and $h_{n, n-i}=\left(2^{n}-1-\cdots-\left(\begin{array}{c}n \\ n-i\end{array}\right)\right)^{3}$ for small $i \geq 0$. This yields the following equation on the sum:

$$
(n+1) H_{n+2}-(7 n+12) H_{n+1}-4(2 n+1) H_{n}+2(10-9 n) 8^{n}=0 .
$$

Finally, solving the previous recurrence by Petkovšek's algorithm (Petkovšek 1992) computes the announced closed form evaluation as a linear combination of hypergeometric terms.

\section{Closure Properties}

The summands and integrands under consideration in the examples of $\S \S 1.4$, 2.1 and 2.4 are products of $\partial$-finite functions. An important point is the possibility to generate the systems which define these functions as $\partial$-finite from a database of "elementary" $\partial$-finite functions. We begin the section by explaining how this can be performed via the closure properties of $\partial$-finite functions. To this end, we appeal once again to Gröbner bases to compute normal forms in quotient rings, and adapt the FGLM algorithm commonly used in the theory of commutative Gröbner bases (Faugère et al. 1993). Next, we illustrate another method for indefinite summation and integration based on a complete elimination and a Gröbner basis calculation for modules.

\subsection{Addition, Product and Derivation of $\partial$-Finite Func- tions by the FGLM Algorithm}

A nice property of $\partial$-finite functions - in fact, one of the main reasons for their introduction in (Chyzak and Salvy 1996) - is their closure under addition, product and derivation. More specifically, assume $f$ and $g$ to be two $\partial$-finite functions with respect to an Ore algebra $\mathbb{O}=\mathbb{F}[\boldsymbol{\partial} ; \boldsymbol{\sigma}, \boldsymbol{\delta}]$. Then $\mathbb{O} \cdot f$ and $\mathbb{O} \cdot g$ are two finite-dimensional $\mathbb{F}$-vector spaces which admit finite bases $\left\{b_{i}\right\}_{i=1, \ldots, N}$ and $\left\{c_{i}\right\}_{i=1, \ldots, M}$, respectively. Then, the derivatives of the sum $f+g$ can be reduced on the generating set constituted of the $b_{i}$ 's and the $c_{i}$ 's. The sum $f+g$ is thus $\partial$-finite. Similarly, the derivatives of the product $f g$ can be reduced on the generating set constituted of the $c_{i} b_{j}$ 's. This entails that the product is also $\partial$-finite. Finally, each derivative $\partial_{k} \cdot f$ is $\partial$-finite, as follows from the reduction of its derivatives on the basis of the $b_{i}$ 's.

Computationally, one usually chooses basis elements of the form $\boldsymbol{\partial}^{\boldsymbol{\alpha}}$, and uses an extension of the FGLM algorithm (Faugère et al. 1993) to take mixed derivatives into account. This extension is described in (Chyzak and Salvy 1996), from which the following example is borrowed. 
We compute annihilators for the sum of the exponential function $f(x, y)=$ $\exp (\mu x+\nu y)$ and of the product of Bessel functions $g(x, y)=J_{\mu}(x) J_{\nu}(y)$. The functions $f$ and $g$ are defined by the systems

$$
\left\{\begin{array} { l } 
{ f _ { x } - \mu f = 0 , } \\
{ f _ { y } - \nu f = 0 }
\end{array} \quad \text { and } \quad \left\{\begin{array}{l}
x^{2} g_{x, x}+x g_{x}+\left(x^{2}-\mu^{2}\right) g=0 \\
y^{2} g_{y, y}+y g_{y}+\left(y^{2}-\nu^{2}\right) g=0
\end{array}\right.\right.
$$

respectively (indices denoting differentiation). First, the algorithm reduces 1 , $D_{y}, D_{x}, D_{y}^{2}, D_{x} D_{y}$ and detects that they are independent. Then $D_{x}^{2}$ is reduced and found to satisfy a linear relation with the previous ones, expressed by the following operator:

$$
\begin{aligned}
p_{1}= & -\left(x^{2}-\mu^{2}+x^{2} \mu^{2}+\mu x\right) y^{2} D_{y}^{2}+x^{2}\left(y^{2}-\nu^{2}+y^{2} \nu^{2}+\nu y\right) D_{x}^{2} \\
& -\left(x^{2}-\mu^{2}+x^{2} \mu^{2}+\mu x\right) y D_{y}+x\left(y^{2}-\nu^{2}+y^{2} \nu^{2}+\nu y\right) D_{x} \\
& -\mu^{2} y^{2} \nu^{2}+x^{2} \nu y+x^{2} y^{2} \nu^{2}-x^{2} \mu^{2} y^{2}+x^{2} \mu^{2} \nu^{2}-\mu x y^{2}+\mu x \nu^{2}-\mu^{2} \nu y .
\end{aligned}
$$

Next, the algorithm continues by reducing $D_{y}^{3}$ and finds another relation

$$
\begin{aligned}
p_{2}= & y^{2}\left(y^{2}-\nu^{2}+\nu y+y^{2} \nu^{2}\right) D_{y}^{3}-y\left(y^{3} \nu+y^{3} \nu^{3}-y^{2}-2 \nu y-\nu^{3} y+3 \nu^{2}\right) D_{y}^{2} \\
& +\left(y^{4}+y^{4} \nu^{2}-y^{3} \nu^{3}-y^{2}-y^{2} \nu^{4}-4 y^{2} \nu^{2}-\nu^{2}+\nu^{4}\right) D_{y} \\
& +\nu\left(-y^{4}-y^{4} \nu^{2}+y^{2}-\nu^{4}+2 y^{2} \nu^{2}+\nu^{2}+y^{2} \nu^{4}-y^{3} \nu+3 \nu^{3} y\right)
\end{aligned}
$$

Finally, the reduction of $D_{x} D_{y}^{2}$ produces the operator

$$
p_{3}=y^{2} D_{x} D_{y}^{2}-\mu y^{2} D_{y}^{2}+y D_{x} D_{y}-\mu y D_{y}+\left(y^{2}-\nu^{2}\right) D_{x}-\mu\left(y^{2}-\nu^{2}\right) .
$$

The system $\left\{p_{1}, p_{2}, p_{3}\right\}$ makes it possible to rewrite any derivative of the sum $f+g$ as a linear combination of 5 derivatives.

\subsection{Indefinite Summation and Integration by Gröbner Bases}

In $\S 1.4$, we looked for the indefinite sum or integral of a function in the module spanned by this function. More specifically, for a $\partial$-finite function $f$ with respect to an Ore algebra $\mathbb{O}$, the method either returns an explicit indefinite sum or integral $F$ in the form $F=Q \cdot f$ for $Q \in \mathbb{O}$ or a proof that no such $F$ exists. In the present section, we turn to the unfavorable case. We no longer search for an explicit form for $F$, but for a system of operators that describes $F$ as a $\partial$-finite function. This is performed by Gröbner basis calculations, as exemplified by the following example borrowed from (Chyzak and Salvy 1996). Informally, the idea is to apply creative telescoping by regarding an indefinite sum or integral as a definite one.

Let $f(x, y)=\left(1+x y+y^{2}\right)^{-2}$. We look for a specification of its indefinite integral $F(x, y)=-\int_{y}^{+\infty} f(x, t) d t$, which is defined for any $x$. Working in the 
Ore algebra $\mathbb{O}=\mathbb{C}(x, y)\left[D_{x} ; 1, D_{x}\right]\left[D_{y} ; 1, D_{y}\right]$, the algorithm of $\S 1.4$ returns a proof that no indefinite integral exists in $\mathbb{O} \cdot f=\mathbb{C}(x, y)$. We thus try another method. The function $f$ is annihilated by both operators

$$
p_{x}=\left(1+x y+y^{2}\right) D_{x}+2 y \quad \text { and } \quad p_{y}=\left(1+x y+y^{2}\right) D_{y}+2 x+2 y,
$$

from which trivially follows that $p_{x} D_{y}$ and $p_{y} D_{y}$ annihilate the indefinite integral $F$. Our goal is to find other operators that annihilate $F$, so as to achieve a $\partial$-finite description. The elimination of $y$ between the polynomials $p_{x}$ and $p_{y}$ in $\mathbb{C}(x)[y]\left[D_{x} ; 1, D_{x}\right]\left[D_{y} ; 1, D_{y}\right]$ yields

$$
P=A D_{y}+B \text { where }\left\{\begin{array}{l}
A=x(x-2)(x+2) D_{x}+x D_{y}+2\left(x^{2}+1\right), \\
B=-x(x-2)(x+2) D_{x}^{2}-4\left(x^{2}+1\right) D_{x}
\end{array}\right.
$$

Creative telescoping in the definite case would go on by the integration of the identity $P \cdot f=0$. Similarly, $h=P \cdot F$ is an indefinite integral of $P \cdot f$ and we immediately have $D_{y} \cdot h=0$, so that $h$ is constant with respect to $y$. Since $F$ and all its cross-derivatives tend to 0 when $y$ tends to $+\infty$, so does $h$. Thus $h=0$, i.e., $A \cdot f+B \cdot F=0$. It then suffices to find a left annihilator for $A$ modulo $\mathrm{Ann}_{\mathbb{O}} f$ to get an operator that annihilates $F$. To compute those $C$ such that $C A=0$ modulo the ideal generated by $p_{x}$ and $p_{y}$ in $\mathbb{O}$, we adapt a method used in the commutative case for the calculation of ideal quotients and based on the calculation of modules of syzygies (Becker and Weispfenning 1993: Algorithm IDEALDIV1). More precisely, we introduce new commutative indeterminates $t, u, v$ and $w$, and eliminate $t$ between the polynomials $u-t A, v-t p_{x}$ and $w-t p_{y}$, by computing a Gröbner basis in the algebra $\mathbb{C}(x, y, u, v, w)[t]\left[D_{x} ; 1, D_{x}\right]\left[D_{y} ; 1, D_{y}\right]$. In this Gröbner basis, those polynomials which do not involve $t$ are of the form $u U+v V+w W$, where $U, V$ and $W$ are polynomials in $\mathbb{O}$ such that $U A+V p_{x}+W p_{y}=0$. Consider the $U$ 's obtained in this way. Their right multiplication by $B$ yields new operators that annihilate $F$ :

$$
\left\{\begin{array}{c}
\left(y\left(x^{3} y+4 x^{2}+4+16 x y+4 x^{2} y^{2}+4 y^{2}\right)\right. \\
\left.+\left(1+x y+y^{2}\right)\left(x^{2} y^{2}+y^{2}+3 x y+1\right) D_{y}\right) \\
\times\left(4\left(x^{2}+1\right) D_{x}+x(x-2)(x+2) D_{x}^{2}\right) \\
\left(32 y^{2}+32 x y+8+48 x^{2} y^{2}+36 x y^{3}+12 x^{3} y^{3}\right) D_{x} \\
+x\left(15 y^{4}+5 x^{2} y^{4}+24 x y^{3}+8 x^{3} y^{3}-2 y^{2}+32 x^{2} y^{2}+28 x y+7\right) D_{x}^{2} \\
+(x-2)(x+2)\left(1+x y+y^{2}\right)\left(x^{2} y^{2}+y^{2}+3 x y+1\right) D_{x}^{3} .
\end{array}\right.
$$

Computing a Gröbner basis from those polynomials adjoined to the ones known beforehand, namely $p_{x} D_{y}$ and $p_{y} D_{y}$, finally yields a basis of a subideal of $\mathrm{Ann}_{\mathbb{O}} F$ constituted of $p_{x} D_{y}, p_{y} D_{y}$ and a third polynomial

$$
\begin{array}{r}
x\left(x^{2}-4\right)\left(1+x y+y^{2}\right) D_{x}^{2}+4\left(x^{2}+1\right)\left(1+x y+y^{2}\right) D_{x} \\
-\left(2 x^{2} y^{2}+2 y^{2}+6 x y+2\right) D_{y} .
\end{array}
$$


This yields a description of $F$ as a $\partial$-finite function.

Note that the method yields a subideal of $\mathfrak{I}=\operatorname{Ann}_{\mathbb{O}} F$ only. Due to the intrinsic weakness in the elimination step of the algorithm, the method may well fail to find the ideal $\mathfrak{I}$, and even a subideal of $\mathfrak{I}$ which describes $F$ as $\partial$-finite. Reasons for this weakness are discussed in the next section.

\section{Dimension and Holonomy}

Intentionally, we have not discussed the termination of the algorithms yet, nor have we justified their success in case of termination. More specifically, all the algorithms for summation and integration in this tutorial are based on the existence of an eliminated polynomial of the form (2.9) or (2.10), and fail or loop forever if no such polynomial exists. In this concluding section, we interpret this existence in terms of ideal dimension, and apply tools based on Gröbner bases to predict the termination of the algorithms, and in some cases decide when to stop them.

The theory of ideal dimension in commutative polynomial algebras extends to a similar theory in Ore algebras. This is the basis for the theory of holonomy (Bernstein 1971, 1972; Björk 1979; Coutinho 1995; Ehlers 1987; Kashiwara $1978)$ in the differential case. Let $\mathbb{O}$ be an Ore algebra $\mathbb{K}[\mathbf{x}][\boldsymbol{\partial} ; \boldsymbol{\sigma}, \boldsymbol{\delta}]$ which satisfies Eq. (1.3) for $c_{i, j}$ 's of total degree at most 1 in $\mathbf{x}$. Let $F_{n}$ be the $\mathbb{K}$-vector space in $\mathbb{O}$ spanned by all terms $\mathbf{x}^{\boldsymbol{\alpha}} \boldsymbol{\partial}^{\boldsymbol{\beta}}$ with total degree $|\boldsymbol{\alpha}|+|\boldsymbol{\beta}|$ at most $n$. Then, for an ideal $\mathfrak{I} \subseteq \mathbb{O}$, the Hilbert function $h(n)=\operatorname{dim}_{\mathbb{K}}\left(F_{n} / \mathfrak{I} \cap F_{n}\right)$ asymptotically agrees with a polynomial called Hilbert polynomial. The degree of this polynomial is called the dimension of the ideal $\mathfrak{I}$. These invariants can be attributed to the module $\mathbb{O} / \mathfrak{I}$ in view of the following generalization to any module. Let $\mathfrak{M}$ be an $\mathbb{O}$-module with generating set $U$, and let $\Gamma_{n}=F_{n} \cdot U$. Then all previous invariants are defined starting from $h(n)=\operatorname{dim}_{\mathbb{K}} \Gamma_{n}$. In the case of an ideal, $U$ is the singleton consisting of the class of 1 modulo $\mathfrak{I}$, and $\Gamma_{n}=F_{n} / \mathfrak{I} \cap F_{n}$. All the previous invariants can be computed from a Gröbner basis for $\mathfrak{I}$ (Bayer and Stillman 1992; Becker and Weispfenning 1993).

In the differential case, the combinatorial description of the dimension of a module has several algebraic and analytic interpretations and is ruled by Bernstein's inequality: when the algebra is generated by $r$ indeterminates $x_{i}$ and $r$ derivation operators $D_{x_{i}}$, Bernstein (1972) proved that the dimension $d$ of a non-zero module $\mathfrak{M}$ satisfies $d \geq r$. A module over the polynomial algebra $\mathbb{O}_{\mathrm{p}}=\mathbb{K}[\mathbf{x}][\boldsymbol{\partial} ; \boldsymbol{\sigma}, \boldsymbol{\delta}]$ is called holonomic when its dimension is precisely the number $r$ of derivation operators in the algebra; a function $f$ in $\mathbf{x}$ is called holonomic when its annihilating ideal $\mathrm{Ann}_{\mathbb{O}_{\mathrm{p}}} f$ is such that the module $\mathbb{O}_{\mathrm{p}} / \mathrm{Ann}_{\mathbb{O}_{\mathrm{p}}} f \simeq \mathbb{O}_{\mathrm{p}} \cdot f$ is holonomic. Moreover, a holonomic function $f$ is $\partial$-finite with respect to the Ore algebra $\mathbb{O}_{\mathrm{r}}=\mathbb{K}(\mathbf{x})[\boldsymbol{\partial} ; \boldsymbol{\sigma}, \boldsymbol{\delta}]$. The converse implication is a deep result due to Kashiwara (1978); see also (Takayama 
1992). For further use of Gröbner bases in relation to holonomy, see (Sturmfels and Takayama 1998).

The motivation for appealing to the theory of holonomy in the context of symbolic summation and integration is that an eliminated operator of the form (2.9) exists as soon as the function is holonomic. More precisely, for any choice of $r-1$ indeterminates from the $2 r$ indeterminates of the algebra, the holonomy of an ideal $\mathfrak{I} \subseteq \mathbb{O}_{\mathrm{p}}$ ensures the existence of a non-zero operator in $\mathfrak{I}$ which does not involve these $r-1$ indeterminates. It follows that the multiple summation and integration of a holonomic function with respect to $r-1$ variables can be performed by a single elimination. See the examples of $\S \S 2.2$ and 2.3 .

To illustrate the combinatorial invariants described above, consider the example of the Appell $F_{4}$ bivariate hypergeometric function, which is obviously $\partial$-finite in view of (0.3). Computing a Gröbner basis for this set of operators in the polynomial algebra $\mathbb{O}_{\mathrm{p}}=\mathbb{Q}(a, b, c, d)[x, y]\left[D_{x} ; 1, D_{x}\right]\left[D_{y} ; 1, D_{y}\right]$ with respect to a term order such that $D_{x} \prec D_{y} \prec x \prec y$ yields a system of 6 operators with respective leading terms $x y^{2} D_{x} D_{y}, x^{2} y D_{x}^{2}, x^{3} D_{x}^{2}, x^{2} y^{2} D_{x}^{3}$, $x y^{3} D_{y}^{4}$ and $y^{4} D_{x}^{2} D_{y}^{3}$. Computing the Hilbert polynomial of the corresponding ideal in $\mathbb{O}_{\mathrm{p}}$ yields $\frac{21}{2} n^{2}-\frac{85}{2} n+73$. This entails that the module $\mathbb{O}_{\mathrm{p}} \cdot F_{4}$ is of dimension 2 , the number of derivation operators in the algebra. The module is thus holonomic, and any of the integration algorithms presented in this tutorial terminates on the input (0.3) computing an annihilating operator for the corresponding integral.

As another example, recall from $\S 1.3$ that the function $e^{\sin z}$ is not $\partial$-finite with respect to the algebra $\mathbb{O}_{\mathrm{r}}=\mathbb{C}(z)\left[D_{z} ; 1, D_{z}\right]$. Correspondingly, the Hilbert polynomial of the module $\mathbb{O}_{\mathrm{p}} \cdot f$ over $\mathbb{O}_{\mathrm{r}}=\mathbb{C}[z]\left[D_{z} ; 1, D_{z}\right]$ is $\frac{n^{2}}{2}+\frac{3 n}{2}+1$ so that the module is of dimension 2 , in particular more than 1 , and the function is not holonomic. The integration of this function is therefore not directly tractable by the algorithms of creative telescoping presented in this tutorial.

As a non-differential example, the function $\left(\begin{array}{l}n \\ k\end{array}\right)$ viewed with respect to the algebra $\mathbb{O}_{\mathrm{p}}=\mathbb{Q}[n, k]\left[S_{n} ; S_{n}, 0\right]\left[S_{k} ; S_{k}, 0\right]$ has an annihilating ideal $\mathfrak{I}$ with Hilbert polynomial $2 n+1$. The corresponding dimension is 1 , which shows that Bernstein's inequality does not hold in general in non-differential Ore algebras. However, the summation of this function is amenable to creative telescoping, provided a description of $\mathfrak{I}$ is given. Indeed, such a summation is always possible as soon as the dimension of the module $\mathbb{O}_{\mathrm{p}} / \mathfrak{I}$ is less than or equal to 2 .

From the examples of this tutorial, it might seem easy to integrate a function $f$ as soon as it known to be holonomic. However, although a description of $f$ as a $\partial$-finite function with respect to $\mathbb{O}_{\mathrm{r}}$ is computationally easy to obtain, a holonomic ideal of annihilating operators in the polynomial Ore algebra $\mathbb{O}_{\mathrm{p}}$ can be really hard to get. An example is provided by the function $f=1 /\left(y^{2}-y+x\right)$. Computing integrals of $f$ with respect to $y$ re- 
quires finding an operator free from $y$ in $\mathbb{O}_{\mathrm{p}}=\mathbb{Q}[x, y]\left[D_{x} ; 1, D_{x}\right]\left[D_{y} ; 1, D_{y}\right]$. The annihilating ideal of $f$ in $\mathbb{O}_{\mathrm{r}}=\mathbb{Q}(x, y)\left[D_{x} ; 1, D_{x}\right]\left[D_{y} ; 1, D_{y}\right]$ is $\mathfrak{K}=$ $\mathbb{O}_{\mathrm{r}} P+\mathbb{O}_{\mathrm{r}} Q$ where $P=D_{y}\left(y^{2}-y+x\right)=\left(y^{2}-y+x\right) D_{y}+(2 y-1)$ and $Q=D_{x}\left(y^{2}-y+x\right)=\left(y^{2}-y+x\right) D_{x}+1$. Any larger ideal in $\mathbb{O}_{\mathrm{r}}$ is $\mathbb{O}_{\mathrm{r}}$ itself. The operator $U=D_{y}^{2}+(4 x-1) D_{x}^{2}+6 D_{x}$ annihilates $f$, so that $U \in \mathfrak{K}$, hence $U \in \mathfrak{K} \cap \mathbb{O}_{\mathrm{p}}$. However, $U$ is not an element of $\mathfrak{I}=\mathbb{O}_{\mathrm{p}} P+\mathbb{O}_{\mathrm{p}} Q$ in $\mathbb{O}_{\mathrm{p}}$. It follows that the algorithm based on a simple elimination of $\S 2.1$ fails to compute any integral, as long as it is given $\mathfrak{I}$ in input. Even worse, the algorithm of $\S 2.3$ fails to terminate. However, if one adjoins the operator $R=\left(y^{2}-y+x\right) D_{y} D_{x}-2 D_{y} \in \mathfrak{K}$, the ideal $\mathbb{O}_{\mathrm{p}} P+\mathbb{O}_{\mathrm{p}} Q+\mathbb{O}_{\mathrm{p}} R \subseteq \mathbb{O}_{\mathrm{p}}$ contains the operator $U$ and both algorithms find it. A similar phenomenon arises when integrating with respect to $x$. On the other hand, the algorithms of $\S 1.4$ and $\S 2.4$ only require the $\partial$-finite description and they succeed in finding the integrals.

\section{Acknowledgements}

Philippe Dumas, Philippe Flajolet, Peter Paule, Bruno Salvy and Berndt Sturmfels read and commented on preliminary versions of this tutorial. In particular, Philippe Dumas and Peter Paule provided me with extremely detailed and impressively accurate comments, which permitted me to improve on the paper significantly. I thank all these contributors most warmly for their precious help.

This work was supported in part by the Long Term Research Project Alcom-IT (\#20244) of the European Union.

\section{References}

Abramov, S. A. (1989a): Problems in computer algebra that are connected with a search for polynomial solutions of linear differential and difference equations. Moscow Univ. Comput. Math. Cybernet., 3:63-68. Transl. from: Vestn. Moskov. univ. Ser. XV Vychisl. mat. kibernet., 3:56-60.

Abramov, S. A. (1989b): Rational solutions of linear differential and difference equations with polynomial coefficients. USSR Computational Mathematics and Mathematical Physics, 29(11):1611-1620. Transl. from: Zh. vychisl. Mat. i mat. Fiz.

Abramov, S. A. (1995): Rational solutions of linear difference and $q$-difference equations with polynomial coefficients. In: Levelt, A. (ed.), Symbolic and algebraic computation (ISSAC '95), Montreal, Canada. ACM Press, New York, pp. 285-289.

Abramov, S. A., Bronstein, M., Petkovšek, M. (1995): On polynomial solutions of linear operator equations. In: Levelt, A. (ed.), Symbolic and algebraic computation (ISSAC '95), Montreal, Canada. ACM Press, New York, pp. 290-296.

Abramov, S. A., Kvashenko, K. Y. (1991): Fast algorithms for the search of the rational solutions of linear differential equations with polynomial coefficients. In: Watt, S. M. (ed.), Symbolic and algebraic computation (ISSAC '91), Bonn, Germany. ACM Press, New York, pp. 267-270. 
Abramov, S. A., Zima, E. V. (1996): A universal program to uncouple linear systems. Preprint.

Almkvist, G., Zeilberger, D. (1990): The method of differentiating under the integral sign. J. Symbolic Comput., 10:571-591.

Andrews, G. E. (1974): An analytic generalization of the Rogers-Ramanujan identities for odd moduli. Proc. Nat. Acad. Sci. U.S.A., 71:4082-4085.

Barkatou, M. A. (1993): An algorithm for computing a companion block diagonal form for a system of linear differential equations. Appl. Algebra Engrg. Comm. Comput., 4:185-195.

Bayer, D., Stillman, M. (1992): Computation of Hilbert functions. J. Symbolic Comput., 14:31-50.

Becker, T., Weispfenning, V. (1993): Groebner bases. Springer-Verlag (Graduate Texts in Mathematics, vol. 141). In cooperation with Heinz Kredel.

Bernstein, I. N. (1971): Modules over a ring of differential operators, study of the fundamental solutions of equations with constant coefficients. Funct. Anal. Appl., 5(2):1-16 (Russian); 89-101 (English translation).

Bernstein, I. N. (1972): The analytic continuation of generalized functions with respect to a parameter. Funct. Anal. Appl., 6(4):26-40 (Russian); 273-285 (English translation).

Björk, J. E. (1979): Rings of Differential Operators. North Holland, Amsterdam.

Bronstein, M., Petkovšek, M. (1994): On Ore rings, linear operators and factorisation. Programmirovanie, 1:27-44. Also available as Research Report 200, Informatik, ETH Zürich.

Bronstein, M., Petkovšek, M. (1996): An introduction to pseudo-linear algebra. Theoret. Comput. Sci., 157(1).

Buchberger, B. (1998): Introduction to Gröbner bases. In the present volume.

Calkin, N. J. (1994): A curious binomial identity. Discrete Math., 131(1-3):335-337.

Chyzak, F. (1997): An extension of Zeilberger's fast algorithm to general holonomic functions. In: Formal Power Series and Algebraic Combinatorics, 9th Conference (FP$S A C$ '97), Vienna, Austria, pp. 172-183.

Chyzak, F., Salvy, B. (1996): Non-commutative elimination in Ore algebras proves multivariate holonomic identities. Research Report 2799, Institut National de Recherche en Informatique et en Automatique. Submitted to: J. Symbolic Comput..

Cohn, P. M. (1971): Free Rings and Their Relations. Academic Press (London Mathematical Society Monographs, number 2).

Coutinho, S. C. (1995): A Primer of Algebraic D-modules. Cambridge University Press (London Mathematical Society Student Texts, number 33).

Cox, D., Little, J., O'Shea, D. (1992): Ideals, Varieties, and Algorithms. An Introduction to Computational Algebraic Geometry and Commutative Algebra. Springer-Verlag, New York.

Ehlers, F. (1987): The Weyl Algebra, volume 2 of Perspectives in Mathematics, chapter 5, pages 173-205. In: Borel, A. et al.: Algebraic D-Modules. Academic Press (Perspectives in Mathematics, vol. 2).

Erdélyi, A. (1981): Higher Transcendental Functions, vol. 1, 2, 3. R. E. Krieger Publishing Company, Malabar, Florida. Second edition. 
Faugère, J., Gianni, P., Lazard, D., Mora, T. (1993): Efficient computation of zerodimensional Gröbner bases by change of ordering. J. Symbolic Comput., 16:329-344.

Galligo, A. (1985): Some algorithmic questions on ideals of differential operators. In: Caviness, B. F. (ed.), Proceedings EUROCAL '85. Springer-Verlag, pp. 413-421 (Lecture Notes in Computer Science, vol. 204).

Gordon, B. (1961): A combinatorial generalization of the Rogers-Ramanujan identities. Amer. J. Math., 83:393-399.

Gosper, R. W. (1978): Decision procedure for indefinite hypergeometric summation. Proc. Nat. Acad. Sci. U.S.A., 75(1):40-42.

Joly, P., Rhaouti, L. (1997): Private communication.

Kandri-Rody, A., Weispfenning, V. (1990): Non-commutative Gröbner bases in algebras of solvable type. J. Symbolic Comput., 9:1-26.

Karr, M. (1981): Summation in finite terms. J. ACM, 28(2):305-350.

Karr, M. (1985): Theory of summation in finite terms. J. Symbolic Comput., 1:303-315.

Kashiwara, M. (1978): On the holonomic systems of linear differential equations II. Invent. Math., 49:121-135.

Kredel, H. (1993): Solvable Polynomial Rings. (Reihe Mathematik), Verlag Shaker, Aachen, Germany, ISBN 3-86111-342-2. After: Kredel, H. (1992): Solvable Polynomial Rings. Doctoral Dissertation, Univ. Passau.

Ore, O. (1933): Theory of non-commutative polynomials. Ann. of Math., 34:480-508.

Paule, P. (1985): On identities of the Rogers-Ramanujan type. J. Math. Anal. Appl., 107(1):255-284.

Paule, P., Riese, A. (1997): A Mathematica $q$-analogue of Zeilberger's algorithm based on an algebraically motivated approach to $q$-hypergeometric telescoping. In: Special functions, $q$-series and related topics, Toronto, Ontario, 1995. Fields Inst. Commun. 14:179-210. Amer. Math. Soc., Providence.

Petkovšek, M. (1992): Hypergeometric solutions of linear recurrences with polynomial coefficients. J. Symbolic Comput., 14:243-264.

Petkovšek, M., Wilf, H., Zeilberger, D. (1996): A=B. A. K. Peters, Wellesley, Massachusset, ISBN 1-56881-063-6.

Prudnikov, A. P., Brychkov, Y. A., Marichev, O. I. (1986): Integrals and Series. Volume 2: Special functions. Gordon and Breach. First edition by Nauka, Moscow (1983).

Risch, R. H. (1969): The problem of integration in finite terms. Trans. Amer. Math. Soc., 139:167-189.

Risch, R. H. (1970): The solution of the problem of integration in finite terms. Bull. Amer. Math. Soc., 76:605-608.

Rogers, L. J. (1894): Second memoir on the expansion of certain infinite products. Proc. London Math. Soc., 25:318-343.

Sturmfels, B., Takayama, N. (1998): Gröbner bases and hypergeometric functions. In the present volume.

Takayama, N. (1989): Gröbner basis and the problem of contiguous relations. Japan J. Appl. Math., 6(1):147-160. 
Takayama, N. (1990a): An algorithm of constructing the integral of a module-an infinite dimensional analog of Gröbner basis. In: Watanabe, S. and Nagata, M. (eds.), Symbolic and algebraic computation (ISSAC '90), Kyoto, Japan. ACM and Addison-Wesley, pp. 206-211.

Takayama, N. (1990b): Gröbner basis, integration and transcendental functions. In: Watanabe, S. and Nagata, M. (eds.), Symbolic and algebraic computation (ISSAC '90), Kyoto. Japan, ACM and Addison-Wesley, pp. 152-156.

Takayama, N. (1992): An approach to the zero recognition problem by Buchberger algorithm. J. Symbolic Comput., 14:265-282.

Trigg, L. (1996): Private communication.

Wegschaider, K. (1997): Computer generated proofs of binomial multi-sum identities. (Diploma Thesis), RISC, Univ. Linz.

Wilf, H. S., Zeilberger, D. (1992a): An algorithmic proof theory for hypergeometric (ordinary and " $q$ ") multisum/integral identities. Invent. Math., 108:575-633.

Wilf, H. S., Zeilberger, D. (1992b): Rational function certification of multisum/integral/ " $q$ " identities. Bull. Amer. Math. Soc., 27(1):148-153.

Zeilberger, D. (1990a): A fast algorithm for proving terminating hypergeometric identities. Discrete Math., 80:207-211.

Zeilberger, D. (1990b): A holonomic systems approach to special functions identities. J. Comput. Appl. Math., 32:321-368.

Zeilberger, D. (1991): The method of creative telescoping. J. Symbolic Comput., 11:195204. 


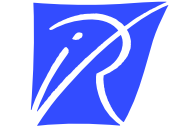

Unité de recherche INRIA Lorraine, Technopôle de Nancy-Brabois, Campus scientifique, 615 rue du Jardin Botanique, BP 101, 54600 VILLERS LĖS NANCY

Unité de recherche INRIA Rennes, Irisa, Campus universitaire de Beaulieu, 35042 RENNES Cedex Unité de recherche INRIA Rhône-Alpes, 655, avenue de l'Europe, 38330 MONTBONNOT ST MARTIN

Unité de recherche INRIA Rocquencourt, Domaine de Voluceau, Rocquencourt, BP 105, 78153 LE CHESNAY Cedex

Unité de recherche INRIA Sophia-Antipolis, 2004 route des Lucioles, BP 93, 06902 SOPHIA-ANTIPOLIS

Cedex

Éditeur

INRIA, Domaine de Voluceau, Rocquencourt, BP 105, 78153 LE CHESNAY Cedex

(France)

http://www.inria.fr

ISSN 0249-6399 